\title{
Dynamics of Abelian Higgs Vortices in the Near Bogomolny Regime
}

\section{Stuart}

Mathematics Department, U.C. Davis, CA95616, USA. email: me@aztec.ucdavis.edu

Received: 30 November 1992

\begin{abstract}
The aim of this paper is to give an analytical discussion of the dynamics of the Abelian Higgs multi-vortices whose existence was proved by Taubes ([JT82]). For a particular value of a parameter of the theory, $\lambda$, called the Higgs self-coupling constant, there is no force between two vortices and there exist static configurations corresponding to vortices centred at any set of points in the plane. This is known as the Bogomolny regime. We will develop some formal asymptotic expansions to describe the dynamics of these multi-vortices for $\lambda$ close, but not equal to, this critical value. We shall then prove the validity of these asymptotic expansions. These expansions allow us to give a finite dimensional Hamiltonian system which describes the vortex dynamics. The configuration space of this system is the "moduli space" - the space of solutions of the static equations modulo gauge equivalence. The kinetic energy term in the Hamiltonian is obtained from the natural metric on the moduli space given by the $L^{2}$ inner product of the tangent vectors. The potential energy gives the intervortex potential which is non-zero when $\lambda$ is not given by its critical value. Thus the reduced equations for the evolution of the vortex parameters take the form of geodesics, with force terms to express the departure from the Bogomolny regime. The geodesics are geodesics on the moduli space with respect to the metric defined by the $L^{2}$ inner product of the tangent vectors, in accordance with Manton's suggestion ([Man82]). This allows an understanding of the two main phenomenological issues - first of all there is the right angle scattering phenomenon, according to which two vortices passing through one another scatter through ninety degrees. Secondly there is the conjecture from numerical calculations that vortices repel for $\lambda$ greater than the critical value, and attract for $\lambda$ less than this value. The results of this paper allow a rigorous understanding of the right angle scattering phenomenon ([Sam92, Hit88]) and reduce the question of attraction or repulsion in the near Bogomolny regime to an understanding of the potential energy term in the Hamiltonian ([JR79]).
\end{abstract}

\section{Introduction}

The aim of this paper is to give an analytical discussion of the dynamics of the Abelian Higgs multi-vortices whose existence was proved by Taubes ([JT82]). The 
model consists of the two dimensional Maxwell equations coupled to a complex scalar field with Ginzburg-Landau type self-interaction:

$$
\begin{aligned}
\nabla \cdot E & =\left(i \Phi, D_{0} \Phi\right), \\
\frac{\partial E_{1}}{\partial t}+\frac{\partial B}{\partial x_{2}} & =\left(i \Phi, D_{1} \Phi\right), \\
\frac{\partial E_{2}}{\partial t}-\frac{\partial B}{\partial x_{1}} & =\left(i \Phi, D_{2} \Phi\right), \\
D_{0}^{2} \Phi-D_{1}^{2} \Phi-D_{2}^{2} \Phi & =\frac{\lambda}{2} \Phi\left(1-|\Phi|^{2}\right) .
\end{aligned}
$$

Here $E, B$ are the time and space components of the field (or curvature) two form

$$
F=\sum_{i=1}^{2} E_{i} d t \wedge d x_{i}+B d x_{1} \wedge d x_{2} .
$$

This is the exterior derivative of the vector potential (or connection) one form $A=A_{0} d t+\sum_{i=1}^{2} A_{i} d x_{i}$, and so we have the relations:

$$
\begin{aligned}
& F_{0 i}=E_{i}=\partial_{t} A_{i}-\partial_{i} A_{0}, \\
& F_{12}=B=\partial_{1} A_{2}-\partial_{2} A_{1} .
\end{aligned}
$$

Complex scalar fields are differentiated covariantly according to the rule

$$
D_{\mu}=\partial_{\mu}-i A_{\mu} .
$$

$E$ is known as the electric field while $B$ is known as the magnetic field. The parameter $\lambda$ is called the Higgs self coupling constant. The equations are invariant under gauge transformations - for any smooth function $\chi(t, x)$, the transformation

$$
\begin{aligned}
A_{0} & \rightarrow A_{0}+\frac{\partial \chi}{\partial t}, \\
A_{i} & \rightarrow A_{i}+\frac{\partial \chi}{\partial x_{i}}, \\
\Phi & \rightarrow \Phi e^{i \chi}
\end{aligned}
$$

takes solutions of Eqs. (1-4) into solutions of Eqs. (1-4). This paper concerns the reduction of this system of partial differential equations to a finite dimensional Hamiltonian system which gives an understanding of vortex dynamics. For all values of $\lambda$ these equations have vortex solutions - these are time independent solutions in which $\Phi$ has a single zero and approaches absolute value one at spatial infinity (see Sect. 2). For the critical value $\lambda=1$, there is no force between two vortices and there exist static configurations corresponding to vortices centred at any set of points in the plane (see [JT82] and Sect. 2). This is the Bogomolny regime. A static solution means one which is time independent, and has vanishing time component of the vector potential $A_{0}=0$. We can write these multi-vortex solutions schematically as

$$
A_{i}=a_{i}\left(x ; Z_{1}, \ldots, Z_{N}\right), \quad \Phi=\phi\left(x ; Z_{1}, \ldots, Z_{N}\right),
$$


where the $Z_{i}$ are arbitrary points in the plane which denote the centres of the vortices - the points at which $\Phi=0$. The space of gauge equivalence classes of $N$ vortices is called the moduli space $M_{N}$. In this paper we will give some formal asymptotic expansions which describe the dynamics of these vortices for $\lambda$ close to, but not equal to, one. We will then prove the validity of these asymptotic expansions - more precisely that there exist solutions to Eqs. (1-4) with $\lambda=1 \pm \varepsilon^{2}$, which are $L^{\infty}$ close, for times $O\left(\frac{1}{\varepsilon}\right)$, to multi-vortices with parameters slowly modulating in the fashion suggested by the asymptotics. The form of the asymptotic expansions is interesting - we obtain, to highest order, equations for the evolution of the parameters which take the form of geodesics on the moduli space with force terms to express the departure from the Bogomolny regime. The tangent space to the moduli space at a given multi-vortex consists of square integrable solutions to the static equations linearised about that multi-vortex. The geodesics are with respect to the metric defined by the $L^{2}$ inner product of the tangent vectors. Thus the asymptotic expansions generalise and rigorise the suggestion of Manton that for $\lambda=1$ the scattering of vortices at low energy should be approximated by geodesics on the moduli space ([Man82]).

The moduli space has been investigated in some detail in [Sam92] and we now discuss this before stating our result carefully. The static multi-vortex solutions of the problem with $\lambda=1$ are determined by the positions of the vortices (the points at which $\Phi=0$ ), and are unchanged by an interchange of any pair of these centres. Thus using complex numbers $Z_{\alpha}$ to designate the centres of the vortices we have a moduli space $M_{N}=S^{N}(\mathbf{C})$, the $N$-fold symmetric product of $\mathbf{C}$. In the case $N=2$ this suggests an interesting behaviour for the scattering of two vortices as follows. Firstly it was suggested in [Hit88] that it is natural to suppose we can take the elementary symmetric functions $P=Z_{1}+Z_{2}, Q=Z_{1} Z_{2}$ as complex co-ordinates on $M_{2}$ - see Sect. 2.1. Assuming this to be so, we take advantage of translation invariance to consider geodesics with $P=0, Z_{1}=-Z_{2}=a$. Then if the two vortices initially approach each other along the real axis, we expect that as the vortices pass through one another $Q$ will change sign from negative to positive. But this will correspond to right angle scattering as $Q=-a^{2}$ changing sign corresponds to $a$ becoming $i a$ - i.e. a $90^{\circ}$ rotation! This is discussed in [Sam92, Hit88], subject to the assumption that $P, Q$ are good co-ordinates, i.e. correspond to square integrable solutions of the linearised equations. It is shown there that the geodesics can be conveniently represented as geodesics on a surface of revolution with the appearance of a smoothed out cone. There is a geodesic which passes over the vertex of the cone which corresponds to the aforementioned right angle scattering. Numerical experiments ([SR88] and [KMR88]) confirm these predictions and also show that the phenomenon is robust to changes in $\lambda$. This paper allows a rigorous analytical understanding of this phenomenon. Another phenomenological issue which the paper allows a partial analytical understanding of is that of the attraction/repulsion of the vortices for $\lambda \neq 1$ - it is conjectured and known numerically from [JR79] that for $\lambda>1$ the vortices repel, and for $\lambda<1$ they attract. The main theorem will hopefully allow this to be understood analytically. We will come back to this in Sect. 6.

We will show in Sect. 2 that indeed $P, Q$ are admissible co-ordinates - i.e. that they give four real co-ordinates $q_{\mu}$ with corresponding square integrable solutions to the linearised equations $n_{\mu}$, which satisfy the condition of being orthogonal to 
the gauge flow (see Eqs. $(28,29)$ for details). We will refer to the $n_{\mu}$ as the zero modes. This allows us to define a metric on $M_{2}$ by

$$
g_{q}(\dot{q}, \dot{q})=\sum_{\mu, v} g_{\mu, v} \dot{q}_{\mu} \dot{q}_{v}=\sum_{\mu, v}\left(n_{\mu}, n_{v}\right)_{L^{2}} \dot{q}_{\mu} \dot{q}_{v}
$$

for $\dot{q} \in T_{q} M_{2}$. We will often suppress the $q$ dependence of $\dot{g}$ when no confusion is likely. We will now state the main result of this paper. It amounts to giving a finite dimensional reduction of the Abelian Higgs equations with $\lambda=1+\imath \varepsilon^{2}, l= \pm 1$ to a Hamiltonian system with Hamiltonian $H: T^{*} M_{2} \rightarrow \mathbf{R}$ given by

$$
H(p, q)=\frac{1}{2} g(p, p)+V(q)
$$

where $p \in T_{q}^{*} M_{2}$ is a momentum conjugate to $\dot{q}$ and is given by

$$
p_{\mu}=\sum_{\nu} g_{\mu \nu} \dot{q}_{v}
$$

where $g: T^{*} M_{2} \times T^{*} M_{2} \rightarrow \mathbf{R}$ is the dual metric given by

$$
g(p, p)=g(\dot{q}, \dot{q})
$$

and the potential energy term $V: M_{2} \rightarrow \mathbf{R}$ is defined by

$$
V(q)=\frac{l}{8} \int_{\mathbf{R}^{2}}\left(1-|\phi|^{2}\right)^{2} .
$$

We shall see that the functions $g_{\mu \nu}$ and $V$ are smooth functions on $M_{2}$, and so it makes sense to consider the Hamiltonian system determined by $H$ :

$$
\frac{d p_{i}}{d \tau}=-\frac{\partial H}{\partial q_{i}} \quad \frac{d q_{i}}{d \tau}=\frac{\partial H}{\partial p_{i}} .
$$

We are interested in reducing the full infinite dimensional PDE to this finite dimensional Hamiltonian system. Our main result gives conditions under which there exist solutions to the Abelian Higgs model which are pointwise close to the static solutions with parameters $q(\tau)$ evolving slowly on the time scale $\tau=\varepsilon t$ according to this Hamiltonian system. Such a finite dimensional reduction of the PDE is possible when the energy is close to the energy of the multi-vortices. The time scale $\tau$ is of course here determined by $\lambda=1+i \varepsilon^{2}$.

Notation. (i) For functions like $\psi=(\tilde{a}, \tilde{\phi}): \mathbf{R}^{2} \rightarrow \mathbf{R}^{2} \oplus \mathbf{C}$ we will use spaces $H^{r, a}$ formed by completing with respect to the inner product

$$
|\psi|_{r, a}=\sum_{|k|=0}^{r} \int_{\mathbf{R}^{2}}\left|\nabla^{k} \tilde{a}\right|^{2}+\left|D_{a}^{k} \tilde{\phi}\right|^{2} .
$$

If we write $|\cdot|_{r, a(q)}$ this will mean differentiation is with respect to the background connection $a(\cdot, q)$. The connection $a$ will be suppressed when no confusion is possible.

(ii) We decompose solutions of the linearised equations $n_{\mu}=\left(n_{\mu}^{1}, n_{\mu}^{2}\right)$ into its $a$ and $\phi$ components in order to write out the initial data.

(iii) For complex numbers we use the usual inner product $(a, b)=(\bar{a} b+a \bar{b}) / 2$. 
(iv) Finally, we state a gauge invariant Sobolev inequality - the problem is how to deal with the covariant derivatives. This is solved by Kato's inequality, which is proved in [JT82]: for a smooth complex valued field $\Phi$ on the plane

$$
\int_{\mathbf{R}^{2}}|\nabla| \Phi||^{2} \leqq \int_{\mathbf{R}^{2}}\left(D_{a} \Phi, D_{a} \Phi\right)
$$

where $a$ is any smooth connection. From this we can deduce covariant versions of the ordinary Sobolev inequalities, in particular for $\psi$ as above

$$
|\psi|_{L^{\infty}\left(\mathbf{R}^{2}\right)} \leqq c|\psi|_{2, a} .
$$

Main Theorem 1. Consider the initial value problem for the Abelian Higgs model, Eqs. (1-4), with $\lambda=1+\imath \varepsilon^{2}$, and initial data which is close to a two vortex $(a(q(0)), \phi(q(0)))$ in the following sense:

$$
\begin{aligned}
A(0, x) & =a(x ; q(0))+\varepsilon^{2} \tilde{a}(0), & A_{t}(0) & =\varepsilon \sum_{\mu} \dot{q}_{\mu}(0) n_{\mu}^{1}+\varepsilon^{2} \tilde{a}_{t}(0), \\
\Phi(0, x) & =\phi(x ; q(0))+\varepsilon^{2} \tilde{\phi}(0), & \Phi_{t}(0, x) & =\varepsilon \sum \dot{q}_{\mu}(0) n_{\mu}^{2}+\varepsilon^{2} \tilde{\phi}_{t}(0),
\end{aligned}
$$

where $(\tilde{a}(0), \tilde{\phi}(0))$ satisfy the conditions in Eqs. 14, 15 and:

$$
|(\tilde{a}(0), \tilde{\phi}(0))|_{3, a(q(0))}+\left|\left(\tilde{a}_{t}(0), \tilde{\phi}_{t}(0)\right)\right|_{2, a(q(0))} \leqq K .
$$
Then for $\varepsilon$ sufficiently small there exists a time $T_{*}=O\left(\frac{1}{\varepsilon}\right)$ such that on the interval
$\left[0, T_{*}\right]$ there exists a solution of the form

$$
\begin{aligned}
\Phi & =\phi(x ; q(t)) e^{i \Xi}+\varepsilon^{2} \tilde{\phi}, \quad A=a(x ; q(t))+d \Xi+\varepsilon^{2} \tilde{a}(t, x), \\
q_{\mu}(t) & =q_{\mu}^{0}(\tau)+\varepsilon \tilde{q}_{\mu}(t), \quad p_{\mu}(t)=p_{\mu}^{0}(\tau)+\varepsilon \tilde{p}_{\mu}(t),
\end{aligned}
$$

where $p^{0}(\tau), q^{0}(\tau)$ are solutions of the Hamiltonian system

$$
\frac{d p_{i}}{d \tau}=-\frac{\partial H}{\partial q_{i}}, \quad \frac{d q_{i}}{d \tau}=\frac{\partial H}{\partial p_{i}},
$$

with $H$ determined by Eq. (6), with initial data

$$
q_{\mu}^{0}(0)=q_{\mu}(0), \quad p_{\mu}^{0}(0)=\sum_{v} g_{\mu \nu} \dot{q}_{v}(0)
$$

where $(\tilde{a}, \tilde{\phi})$ satisfy the conditions

$$
\begin{gathered}
\nabla \cdot \tilde{a}(t, \cdot)-(i \varphi(\cdot ; q(t)), \tilde{\phi}(t, \cdot))=0, \\
\left(n_{\mu},(\tilde{a}(t), \tilde{\phi}(t))\right)=0,
\end{gathered}
$$

and the maps

$$
\begin{aligned}
& t \rightarrow \tilde{p}(t), \quad t \rightarrow \frac{1}{\varepsilon} \frac{d p}{d t}, \\
& t \rightarrow \tilde{q}(t), \quad t \rightarrow \frac{1}{\varepsilon} \frac{d q}{d t}, \\
& t \rightarrow\left((\tilde{a}, \tilde{\phi}),\left(\tilde{a}_{t}, \tilde{\phi}_{t}\right)\right) \in H^{3, a(q(0))} \oplus H^{2, a(q(0))}
\end{aligned}
$$

are continuous and bounded independent of $\varepsilon$. In addition $\left|A_{0}(t, \cdot)\right|_{L^{\infty}\left(\mathbf{R}^{2}\right)}=O\left(\varepsilon^{3}\right)$, the map $t \rightarrow \Xi(t) \in C^{\infty}\left(\mathbf{R}^{2}\right)$ is twice differentiable and the solution has regularity

$$
(\tilde{q}, \tilde{p}) \in C^{2}\left(\left[0, T_{*}\right]\right) \oplus C^{1}\left(\left[0, T_{*}\right]\right)
$$


and

$$
(\tilde{a}, \tilde{\phi}) \in C^{1}\left(\left[0, T_{*}\right], H^{1, a} \oplus L^{2}\right) \cap C\left(\left[0, T_{*}\right], H^{3, a} \oplus L^{2}\right) .
$$

The additional conditions on the initial data we need are:

$$
\begin{gathered}
\left(n_{\mu},(\tilde{a}(0), \tilde{\phi}(0))\right)=0,\left.\quad \frac{d}{d t}\right|_{t=0}\left(n_{\mu},(\tilde{a}, \tilde{\phi})\right)=0, \\
\nabla \cdot \tilde{a}(0, \cdot)-(i \varphi(\cdot ; q(0)), \tilde{\phi}(0, \cdot))=0 .
\end{gathered}
$$

Remarks. (i) The conditions in Eqs. (12) and (15) are required in order to "fix the gauge" - the solutions are only expected to be unique up to gauge equivalence. The function $\Xi(t)$ is a gauge transformation of the base multi-vortex solution, and has no dynamical significance. It is chosen in such a way that $\frac{d}{d t}\left(a+d \Xi, \Phi e^{i \Xi}\right)$ satisfies the condition in Eq. (12). The geometric meaning of these conditions is explained in Sect. 2. (ii) The conditions in Eq. (13) are more physically meaningful, as we now explain. Imagine that we are given a solution at time $t, A(t, \cdot), \Phi(t, \cdot)$ and want to decide which multi-vortex to which it is closest. An obvious thing to do ([Ben72]) is to minimise the following distance function over all the vortex configurations:

$$
\min _{Z_{1}, Z_{2}}\left(\left|A(t, \cdot)-a\left(\cdot ; Z_{1}, Z_{2}\right)\right|_{L^{2}}^{2}+\left|\Phi(t, \cdot)-\phi\left(\cdot ; Z_{1}, Z_{2}\right)\right|_{L^{2}}^{2}\right) .
$$

Formally speaking we would hope that at the minima we would have the following orthogonality conditions, obtained as the differential conditions for a minimum:

$$
\left(A(t, \cdot)-a\left(\cdot, Z_{1}, Z_{2}\right), \frac{\partial a}{\partial Z_{i}}\right)+\left(\Phi(t, \cdot)-\phi\left(\cdot, Z_{1}, Z_{2}\right), \frac{\partial \phi}{\partial Z_{i}}\right)=0 .
$$

Now since $(a(\cdot ; Z), \phi(\cdot ; Z))$ are solutions of the full static equations for all $Z$, we expect that the derivatives $\frac{\partial a}{\partial Z_{i}}, \frac{\partial \phi}{\partial Z_{i}}$ should be solutions of the linearised equations. These derivatives are thus candidates for the zero modes $n_{\mu}$. In fact this is not quite right because the derivatives $\frac{\partial a}{\partial Z_{i}}, \frac{\partial \phi}{\partial Z_{i}}$ are not square integrable - this is explained fully in Sect. 2. Nevertheless we shall see in Sect. 4 that on account of condition (12) we can think of the conditions in (13) as giving the parameters of the multi-vortex closest to the solution at time $t$. These same conditions thus determine the time dependence of the vortex parameters. In other words the modulation of the parameters is such as to minimise the $L^{2}$ distance of the solutions from the corresponding multi-vortex. This is a general feature of solitary wave perturbation theory, and the reader may wish to take a look at [Ben72], [DHW82], [MS78] and [Stu] to see the asymptotic situation in somewhat simpler situations. In particular in [Stu] it is shown how to generalise the approximations of Manton ([Man82]) for self-dual monopole dynamics to more general situations. However a rigorous argument has not yet been given for this case. ${ }^{1}$

(iii) The basis for the proof of the theorem is to use conservation of energy to estimate the errors. At the level of the error terms $(\tilde{a}, \tilde{\phi})$ the energy of the Abelian

\footnotetext{
${ }^{1}$ This has since been successfully carried out in [D.S]
} 
Higgs model is replaced by its Hessian. We will see in Sect. (3) that the Higgs mechanism, which physically gives the particles of the quantum theory mass, has the effect of pushing the essential spectrum away from the origin, thus making the Hessian equivalent to the $H^{1}$ norm on the subspace orthogonal to the zero modes $n_{\mu}$. This provides the method of proof.

(iv) The method of proof leads to a coupled system of ordinary, elliptic and hyperbolic differential equations. A local existence result for this system is given in the appendix which is proved by a simple iteration scheme. The result which this gives is probably not optimal, and so the regularity statement in the statement of the main theorem could not presumably be improved. The solutions are expected to be unique up to gauge equivalence, a fact which could be proved by standard methods.

We start off by reviewing the static Abelian Higgs model, then prove a few new results about this which we will need for our work, before proceeding to the dynamical situation.

\section{Review of the Static Abelian Higgs Model}

In this part of the paper we discuss the Abelian Higgs model, which is the two dimensional gauge theory corresponding to the circle group $S^{1}$ coupled to a Higgs field with Ginzburg-Landau type self-interaction. The energy of the theory is

$$
\mathscr{E}=\frac{1}{2} \int_{\mathbf{R}^{2}} E^{2}+B^{2}+\left|D_{A} \Phi\right|^{2}+\frac{\lambda}{4}\left(1-|\Phi|^{2}\right)^{2}
$$

where $E_{i}=\partial_{t} A_{i}-\partial_{i} A_{0}$ is the electric field, $B=\partial_{1} A_{2}-\partial_{2} A_{1}$ is the magnetic field, and $D_{A}$ is the covariant derivative $D_{A} \Phi=d \Phi-i A \Phi$ with respect to the connection $A$. The connection will be suppressed when there is no possibility of confusion. We shall be interested in the time-dependent problems corresponding to modulating static solutions, so we shall start off by recalling some facts about the static solutions from [JT82]. The static version of the theory corresponds to time independent configurations with $A_{0}=0$, which minimise the energy (subject to boundary conditions at spatial infinity). This gives rise to the equations:

$$
\begin{gathered}
-\partial_{1}^{2} A_{2}+\partial_{1} \partial_{2} A_{1}+\frac{i}{2}\left(\bar{\Phi} D_{1} \Phi-\Phi D_{1}^{-} \Phi\right)=0 \\
-\partial_{2}^{2} A_{2}+\partial_{1} \partial_{2} A_{2}+\frac{i}{2}\left(\bar{\Phi} D_{2} \Phi-\Phi D_{2}^{-} \Phi\right)=0 \\
-D_{1}^{2} \Phi-D_{2}^{2} \Phi=\frac{\lambda}{2} \Phi\left(1-|\Phi|^{2}\right)
\end{gathered}
$$

which are invariant under gauge transformations

$$
A \rightarrow A+d \chi, \quad \Phi \rightarrow \Phi e^{i \chi}
$$

for any real valued smooth function $\chi$ on the plane. These equations have a very interesting behaviour as $\lambda$ varies. The best way to see this is the Bogomolny 
decomposition of the energy functional:

$$
\begin{aligned}
\mathscr{E}= & \int_{\mathbf{R}^{2}}\left|\left(\partial_{1} \Phi_{1}+A_{1} \Phi_{2}\right) \mp\left(\partial_{2} \Phi_{2}-A_{2} \Phi_{1}\right)\right|^{2}+\left|\left(\partial_{2} \Phi_{1}+A_{2} \Phi_{2}\right) \pm\left(\partial_{1} \Phi_{2}-A_{1} \Phi_{1}\right)\right|^{2} \\
& +\left|B \pm \frac{1}{2}\left(\Phi_{1}^{2}+\Phi_{2}^{2}-1\right)\right|^{2} \pm B+\frac{(\lambda-1)}{4}\left(1-|\Phi|^{2}\right)^{2} .
\end{aligned}
$$

Thus we see that if $\lambda=1$ then the energy decomposes into positive definite terms, plus the term $\int_{\mathbf{R}^{2}} B=\lim _{R \rightarrow \infty} \int_{|x|=R} A \cdot d x$, which depends only on the boundary behaviour. Thus if we minimise with $\int_{\mathbf{R}^{2}} B$ fixed we obtain the Bogomolny equations

$$
\begin{gathered}
\left(\partial_{1} \Phi_{1}+A_{1} \Phi_{2}\right) \mp\left(\partial_{2} \Phi_{2}-A_{2} \Phi_{1}\right)=0, \\
\left(\partial_{2} \Phi_{1}+A_{2} \Phi_{2}\right) \pm\left(\partial_{1} \Phi_{2}-A_{1} \Phi_{1}\right)=0, \\
B \pm \frac{1}{2}\left(\Phi_{1}^{2}+\Phi_{2}^{2}-1\right)=0 .
\end{gathered}
$$

We shall describe the consequences of this after discussing the boundary term $\int_{\mathbf{R}^{2}} B$, which has a topological significance as the winding number of the Higgs field $\Phi$. Indeed in [JT82] it is proved that if $\Phi, A$ are continuous functions on the plane such that

(i) $\lim _{R \rightarrow \infty} \sup _{|x|=R}|1-| \Phi||=0$,

(ii) $|x|^{1+\delta}|D \Phi| \leqq$ const,

(iii) $B \in L^{1}$,

and we define $e=\Phi /|\Phi|$, then

$$
\int_{\mathbf{R}^{2}} B=\lim _{R \rightarrow \infty} \int_{|x|=R} A \cdot d x=\frac{-i}{4 \pi} \lim _{R \rightarrow \infty} \int(\bar{e} d e-e d \bar{e})
$$

is an integer, the winding number or vortex number of the scalar field $\Phi$.

To explain the phrase vortex number we now discuss vortex solutions. It is known (see e.g. [Plo80]), that Eqs. (17-19) have solutions with radial symmetry, which means that using radial co-ordinates they can be expressed as

$$
\Phi=u(r) e^{i \theta}, \quad A=a(r) d \theta .
$$

These are called vortices, and correspond to vortex number +1 . Computational studies of [JR79] suggest that if $\lambda>1$ two vortices will repel while for $\lambda<1$ they attract and the net force is zero for $\lambda=1$. This is because the repulsion of the magnetic field cancels the attraction of the complex scalar field for $\lambda=1$. This gives rise to the possibility of there existing multi-vortices at this value. Taubes proved that this is indeed the case (see [JT82]). We shall now describe how the Bogomolny decomposition and consequent reduction of Eqs. (17-19) to the first order form of Eqs. (22-24) allows a verification of this critical behaviour. We minimise $\mathscr{E}$ subject to the condition of fixed positive winding number $N$, so we are led to Eqs. (22-24) with the upper signs. It turns out that these equations are equivalent to a single second order equation for a scalar, a method which goes back to [Wit77]. The scalar equation is

$$
-\Delta u+e^{u}-1=-4 \pi \sum \delta_{Z_{\alpha}}
$$


where the $Z_{\alpha}$ are arbitrarily chosen points in the plane, and correspond to the centres of the vortices (zeros of the Higgs field). The solution of Eq. (25) generates solutions of Eqs. (22-24) via

$$
\begin{gathered}
\phi=\exp \frac{1}{2}[u+i \Theta], \\
a_{1}=\frac{1}{2}\left(\partial_{2} u+\partial_{1} \Theta\right), \quad a_{2}=-\frac{1}{2}\left(\partial_{1} u-\partial_{2} \Theta\right),
\end{gathered}
$$

where we choose $\Theta=2 \sum \arg \left(z-Z_{\alpha}\right.$ ). (By Eq. (20) there are also gauge equivalent solutions.) To see why the points $\left\{Z_{\alpha}\right\}$ are referred to as the centres of the vortices, we look at the appearance of the solutions close to these points. The delta sources in Eq. (25) enforce the behaviour $u \sim 2 \ln \left|z-Z_{\alpha}\right|$, which means that $\Phi \sim\left(z-Z_{\alpha}\right)$ (assuming $Z_{\alpha}$ appears only once in the list of vortex centres). Solutions of Eq. (25) thus generate what can sensibly be called multi-vortex solutions of Eqs. (17-19) when $\lambda=1$. We now state Taubes' existence result ([JT82]).

Theorem 2.1 (Existence). Every critical point of the functional Eq. (16) with $\lambda=1$ and $\int_{\mathbf{R}^{2}} B=N \in \mathbf{N}$ is a solution of the first order equations (22-24). Each such solution a, $\phi$ is determined, modulo gauge equivalence, by a set of $N$ points in the plane $Z_{\alpha}$, and is given by Eqs. (25-27). Furthermore the solution is smooth, and has the following properties:

(a) $\phi \sim c_{j}\left(z-Z_{j}\right)^{n_{j}}$ as $z \rightarrow Z_{j}$, where $Z_{j}$ occurs $n_{j}$ times.

(b) Let the $\left\{Z_{j}\right\}$ be contained in some compact set $K$. Then for any $\delta>0$ there exists $c(\delta, K)$ such that

$$
|D \phi|,(1-|\phi|) \leqq \mathrm{c}(\delta) \mathrm{e}^{-(1-\delta)|\mathrm{z}|}
$$

(c) $\mathscr{E}=\pi N=\frac{1}{2} \int_{\mathbf{R}^{2}} B=\pi \sum n_{j}$.

Similar results hold for $N<0$.

In calculating the dynamics of these multi-vortex solutions a prominent role is played by the solutions to the linearised equations (zero modes), which span the tangent space to the moduli space. The moduli space is the space of gauge equivalence classes

$$
(\phi, a) \sim\left(\phi e^{i \chi}, a+d \chi\right) .
$$

Thus for our problem the moduli space is $S^{N}(\mathbf{C})$, the symmetric product of $N$ copies of $\mathbf{C}$, because the solution of Eq. (25) is unchanged by the interchange of two vortex centres. It is convenient to introduce the elementary symmetric products of the $Z_{\alpha}$, which are the coefficients $s_{i}$ of the polynomial

$$
p=\sum s_{i} z^{i}=\prod_{\alpha=1}^{N}\left(z-Z_{\alpha}\right)
$$

whose roots are $Z_{\alpha}$. We will find that these are good co-ordinates on $M_{N}$ - see [Hit88] and [Sam92]. To see why this is so we have to discuss in some detail the tangent space.

The tangent space is naturally regarded as being orthogonal to the infinitesimal gauge transformations $(d \chi, i \chi \phi)$ at $(a, \phi)$ :

$$
\int(\tilde{a}, d \chi)+(\tilde{\phi}, i \chi \phi)=0
$$


or equivalently

$$
\nabla \cdot \tilde{a}-(i \phi, \tilde{\phi})=0 .
$$

This will be referred to as the gauge orthogonality condition. Thus we define the tangent space $T_{(a, \phi)}$ to $M_{N}$ at the multi-vortex $(a, \phi)$ as the space of $L^{2}$ functions $(\tilde{a}, \tilde{\phi})$ which satisfy the condition in Eq. (29) together with the linearised Bogomolny equations. These two conditions can be economically written as

where

$$
\mathscr{D}_{(a, \phi)}\left(\begin{array}{c}
\tilde{\alpha} \\
\tilde{\phi}
\end{array}\right)=\left(\begin{array}{c}
\partial \overline{\tilde{\alpha}}+\frac{i}{4} \bar{\phi} \tilde{\phi} \\
\partial \tilde{\phi}-i \bar{\alpha} \tilde{\phi}-i \tilde{\bar{\alpha} \phi}
\end{array}\right)=\left(\begin{array}{l}
0 \\
0
\end{array}\right),
$$

$$
\alpha=\frac{1}{2}\left(a_{1}-i a_{2}\right), \quad \tilde{\alpha}=\frac{1}{2}\left(\tilde{a}_{1}-i \tilde{a}_{2}\right)
$$

and

$$
\bar{\partial}=\frac{1}{2}\left(\partial_{1}+i \partial_{2}\right), \quad \partial=\frac{1}{2}\left(\partial_{1}-i \partial_{2}\right) .
$$

Here the gauge orthogonality condition appears naturally as the real part of the linearised equations written in complex co-ordinates. We now use Eq. (25) to write down a basis for $T_{(a, \phi)}$. For simplicity we consider the two vortex situation $N=2$, and assume for the moment the two vortices are not coincident: $Z_{1} \neq Z_{2}$. Then the obvious way to try to generate solutions to the linearised equations is to differentiate with respect to the parameters, i.e. to consider

$$
\left(\frac{\partial a}{\partial Z_{\alpha, i}}, \frac{\partial \phi}{\partial Z_{\alpha, i}}\right)
$$

for $\alpha=1 \ldots N$ and $i=1,2$ representing the co-ordinate axes, i.e. $Z_{\alpha}=Z_{\alpha, 1}+i Z_{\alpha, 2}$. A quick look at the asymptotic behaviour makes it clear that these are not square integrable. However there is another difficulty - they do not satisfy the gauge orthogonality condition Eq. (29). These two problems cancel out - in other words we can find an infinitesimal gauge transformation $\left(d \tilde{\chi}_{\alpha, i}, i \tilde{\chi}_{\alpha, i} \phi\right)$ such that

$$
\left(\frac{\partial a}{\partial Z_{\alpha, i}}+d \tilde{\chi}_{\alpha, i}, \frac{\partial \phi}{\partial Z_{\alpha, i}}+i \tilde{\chi}_{\alpha, i} \phi\right)
$$

is square integrable, and satisfies both Eq. (29) and Eq. (30). Direct substitution into Eq. (29) leads to the following equation for $\chi_{\alpha, i}$ :

$$
-\Delta \chi_{\alpha, i}+|\phi|^{2} \chi_{\alpha, i}=-|\phi|^{2} \frac{\partial \Theta}{\partial Z_{\alpha, i}}, \quad \Theta=2 \sum \arg \left(z-Z_{\alpha, i}\right) .
$$

Referring to Eqs. (25) and (26) we see that the right-hand is in fact smooth which will allow us to find a smooth solution to this equation. The next results show that the multi-vortex solutions $a(x ; Z), \phi(x ; Z)$ are smooth functions of the parameters $Z_{\alpha, i}$, and the expressions (31) define smooth square integrable solutions of (30) which depend smoothly on the $\left\{Z_{\alpha, i}\right\}$. 
Lemma 2.2. The multivortex solutions are smooth functions of the parameters $Z_{\alpha, i}$.

Lemma 2.3. Equation (32) has a smooth solution which approaches $-\frac{\partial \Theta}{\partial Z_{\alpha, i}}$ as $|z| \rightarrow \infty$ exponentially fast, which has the consequence that:

$$
\int_{\mathbf{R}^{2}}\left(\frac{\partial \phi}{\partial Z_{\alpha, i}}+i \phi \chi_{\alpha, i}\right)^{2}+\left(\frac{\partial a_{j}}{\partial Z_{\alpha, i}}+d_{j} \chi_{\alpha, i}\right)^{2}<\infty .
$$

Corollary 2.4. If $Z_{\alpha} \neq Z_{\beta}$ for $\alpha \neq \beta$ we have $2 N$ independent solutions of $E q$. (30) which are square integrable and are given by

$$
n_{\alpha, i}=\left(\frac{\partial \phi}{\partial Z_{\alpha, i}}+i \phi \chi_{\alpha, i}, \frac{\partial a_{j}}{\partial Z_{\alpha, i}}+d_{j} \chi_{\alpha, i}\right) \text {. }
$$

Furthermore the functions $\left(\frac{\partial \phi}{\partial Z_{\alpha, i}}+i \phi \chi_{\alpha, i}\right),\left(\frac{\partial a_{j}}{\partial Z_{\alpha, i}}+d_{j} \chi_{\alpha, i}\right)$ are smooth functions of the $Z_{\alpha, i}$, with the property that if the $\left\{Z_{\alpha}\right\}$ are contained in some compact set $K$, then for any positive integer $r$, and any number $\delta>0$ there exists $c(\delta, r, K)$ such that

$$
\left|D^{m}\left(\frac{\partial \phi}{\partial Z_{\alpha, i}}+i \phi \chi_{\alpha, i}\right)\right|+\left|D^{m}\left(\frac{\partial a_{j}}{\partial Z_{\alpha, i}}+d_{j} \chi_{\alpha, i}\right)\right| \leqq c(\delta, r, K) e^{-(1-\delta)|x|},
$$

where $D$ represents differentiation with respect to $x_{1}, x_{2}$, and the $Z_{\alpha, i}$, and $m$ is a multi-index with $|m| \leqq r$.

Remark. In this lemma the families of multivortices do not have to be exactly those given by Eqs. (26) and (27) but are allowed to differ by a smooth family of gauge transformations.

Proof of Lemma 2.2. For the proof of this lemma we recall some aspects of the existence proof in [JT82]. The function $u$ which solves (25) is obtained by subtracting out the singularities as follows:

$$
u=u_{0}+v
$$

where

$$
u_{0}=\sum_{\alpha=1}^{N} \ln \left\{\frac{\left|z-Z_{\alpha}\right|^{2}}{\mu+\left|z-Z_{\alpha}\right|^{2}}\right\},
$$

where $\mu>4 N$. This leads to the following equation for the regular part $v$ :

$$
-\Delta v+e^{u_{0}} e^{v}=1-g_{0}, \quad g_{0}=4 \sum_{\alpha=1}^{N} \frac{\mu}{\left(\mu+\left|z-Z_{\alpha}\right|^{2}\right)^{2}} .
$$

It is now clear that to prove the lemma all we have to do is to prove that $v$ is a smooth function of the $Z_{\alpha}$. We will show here that it is $C^{1}-$ the fact that it smooth will then follow either from repeating these arguments - see the proof of Lemma 2.3 and its corollary. To see that $v$ is differentiable we look at the natural candidate for $\frac{\partial v}{\partial Z_{\alpha, i}}$, which is the solution $v_{\alpha, i}^{\prime}$ of the equation:

$$
-\Delta v_{\alpha, i}^{\prime}+e^{u_{0}} e^{v} v_{\alpha, i}^{\prime}=-\frac{\partial g_{0}}{Z_{\alpha, i}}-e^{u_{0}} e^{v} \frac{\partial u_{0}}{\partial Z_{\alpha, i}}
$$



This is easily seen to be smooth and square integrable. To see that it is indeed $\frac{\partial v}{\partial Z_{\alpha, i}}$
we have to show that

$$
w=\frac{v\left(Z_{\alpha, i}+h\right)-v\left(Z_{\alpha, i}\right)-h v_{\alpha, i}^{\prime}}{h}
$$

has limit zero as $h \rightarrow 0$. But $w$ solves an equation of the form

$$
-\Delta w+e^{u_{0}} e^{v} w=h w F\left(v\left(Z_{\alpha, i}+h\right), v\left(Z_{\alpha, i}\right), x\right),
$$

where $F$ is a smooth function, uniformly bounded for $h<1$. Now since we know that $e^{u_{0}+v} \geqq 0$ and $e^{u_{0}+v} \rightarrow 1$ as $|x| \rightarrow \infty$ we have an estimate

$$
\int|\nabla w|^{2}+e^{u_{0}+v}|w|^{2} \geqq \gamma \int|w|^{2}+|\nabla w|^{2}
$$

for sine $\gamma>0$. Thus if we choose $h$ sufficiently small we can see that $|w|_{H^{1}} \rightarrow 0$ as $h \rightarrow 0$. Substitution of this into the equation then shows that $|\Delta w|_{L^{2}} \rightarrow 0$ as $h \rightarrow 0$. It then follows from Sobolev's theorem that $v_{\alpha, i}^{\prime}$ is the classical derivative. We can repeat this process to show that the $\operatorname{map} Z \rightarrow v \in C^{\infty}\left(\mathbf{R}^{2}\right)$ is infinitely differentiable.

Proof of Lemma 2.3. Let $\rho$ be a smooth function equal to one outside a disc $|x|>R_{2}$ and equal to zero inside $|x|<R_{1}<R_{2}$. Then $v_{\alpha, i}=\chi_{\alpha, i}-\rho \frac{\partial \Theta}{\partial Z_{\alpha, i}}$ satisfies an equation of the form

$$
-\Delta v_{\alpha, i}+|\phi|^{2} v_{\alpha, i}=g_{\alpha, i}
$$

where $g_{\alpha, i}$ is smooth and vanishes outside $|x|>R_{2}$ and so is square integrable. Using the fact stated above that $|\phi|$ approaches 1 exponentially fast, it follows that this equation has a solution which decays to zero exponentially, i.e. there are constants $C, \delta$ such that

$$
\left|v_{\alpha, i}\right| \leqq C e^{-\delta x} .
$$

This follows by comparison methods - see Sect. 3.7 in [JT82]. It follows that $\chi_{\alpha, i}$ is our required solution. To see that it depends smoothly on the parameters we have to show that $v_{\alpha_{i}}$ does. This is easily seen as follows - if $\frac{\partial v_{\alpha, i}}{Z_{\alpha, i}}$ exists it should be the solution $h$ of the equation

$$
-\Delta h+e^{u_{0}} h=\frac{\partial g_{\alpha, i}}{Z_{\beta, j}}-v_{\alpha, i} \frac{\partial\left(e^{u_{0}}\right)}{Z_{\beta, j}} .
$$

This is easily seen to exist and indeed to be a derivative. This process can be continued indefinitely to produce any number of derivatives.

Proof of Corollary. First of all we see that $\frac{\partial \phi}{\partial Z_{\alpha, i}}+i \phi \chi_{\alpha, i}$ is smooth and decays exponentially to zero. The term $\frac{\partial a}{\partial Z_{\alpha, i}}+d \chi_{\alpha, i}$ is a sum of exponentially decaying functions and functions of order $r^{-2}$ at infinity - see $(26,27)$. The non-exponentially decaying terms cancel, out leaving an exponentially decaying remainder, which is of course square integrable. The linear dependence of these solutions is easily seen to reduce to the linear dependence of the $\frac{\partial u}{\partial Z_{\alpha, i}}$ from Eq. (25). But any linear 
combination of these

$$
l=\sum c_{\alpha, i} \frac{\partial u}{\partial Z_{\alpha, i}}
$$

satisfies the equation

$$
-\Delta l+e^{u} l=\sum c_{\alpha, i} \frac{\partial}{\partial Z_{\alpha, i}} \delta_{Z_{\alpha, i}},
$$

so $l=0$ only if there is a cancellation of sources which requires $Z_{\alpha}=Z_{\beta}$, contrary to assumption. The fact that the derivatives are exponentially decaying follows by looking at (26) and (27) and noticing that non-exponentially decaying terms cancel out for all derivatives.

2.1. Co-ordinates on the Moduli Space. We now discuss co-ordinates on the moduli space. We have displayed $2 N$ square integrable solutions to Eq. (30) for non-coincident vortices $-Z_{\alpha} \neq Z_{\beta}$. A formal index calculation of Weinberg ([Wei79]) suggests that there should indeed be $2 N$ such zero modes, so that at points where no vortices coincide we say the $Z_{\alpha}$ form a good co-ordinate system. What about points of coincidence? First of all recall that the $N$ vortex moduli space is naturally regarded as $M_{N}=S^{N} \mathrm{C}$. We noted above that the symmetric product $S^{N}(\mathbf{C})$ has as a natural set of co-ordinates the elementary symmetric functions, i.e. the coefficients $s_{i}\left(Z_{\alpha}\right)$ of the polynomial

$$
\prod_{\alpha=1}^{N}\left(z-Z_{\alpha}\right)=\sum_{i=0}^{N} s_{i} z^{i}, \quad s_{N}=1 .
$$

We now show that these give good co-ordinates in the case $N=2$, i.e. they generate square integrable solutions to Eq. (30) for all positions of the vortices including the case $Z_{1}=Z_{2}$. So we introduce

$$
P=Z_{1}+Z_{2}=P_{1}+i P_{2}, \quad Q=Z_{1} Z_{2}=Q_{1}+i Q_{2},
$$

and show that the real and imaginary parts give four independent square integrable solutions of Eq. (30) even when $Z_{1}=Z_{2}$. First of all:

$$
\begin{gathered}
\frac{\partial}{\partial Q}=\frac{1}{Z_{2}-Z_{1}}\left(\frac{\partial}{\partial Z_{1}}-\frac{\partial}{\partial Z_{2}}\right), \\
\frac{\partial}{\partial P}=\frac{1}{Z_{1}-Z_{2}}\left(Z_{1} \frac{\partial}{\partial Z_{1}}-Z_{2} \frac{\partial}{\partial Z_{2}}\right) .
\end{gathered}
$$

Clearly for $Z_{1} \neq Z_{2}$ the real and imaginary parts of these give rise to four independent square integrable solutions of (30), by the previous result. We now propose to examine the limit as $Z_{1} \rightarrow Z_{2}$ of the solutions of Eq. (30) suggested by these. We shall see that they extend to independent solutions at the points of co-incidence.

Lemma 2.5. The operators $\frac{\partial}{\partial Q}, \frac{\partial}{\partial P}$ give rise to four square integrable solutions of $E q$. (30), which extend continuously to $Z_{1}=Z_{2}$, as follows. We write Eqs. $(34,35)$ in 
real terms:

$$
\begin{aligned}
& \frac{\partial}{\partial q}=\sum \sum\left(a_{\alpha, i}+i b_{\alpha, i}\right) \frac{\partial}{\partial Z_{\alpha, i}}, \\
& \frac{\partial}{\partial p}=\sum \sum\left(c_{\alpha, i}+i d_{\alpha, i}\right) \frac{\partial}{\partial Z_{\alpha, i}} .
\end{aligned}
$$

Then the corresponding linear combinations

$$
\begin{aligned}
& n_{Q_{1}}=\sum \sum a_{\alpha, i} n_{\alpha, i}, \quad n_{Q_{2}}=\sum \sum b_{\alpha, i} n_{\alpha, i}, \\
& n_{P_{1}}=\sum \sum c_{\alpha, i} n_{\alpha, i}, \quad n_{P_{2}}=\sum \sum d_{\alpha, i} n_{\alpha, i}
\end{aligned}
$$

are square integrable linearly independent solutions of $E q$. (30) (even for $Z_{1}=Z_{2}$ ). Furthermore all these solutions depend smoothly on $P, Q$, and for $P, Q$ in some compact set $K$ on $M_{2}$, and for any integer, and any number $\delta>0$ there exists $c(K, r, \delta)$ such that

$$
\left|D^{m} n_{\mu}(x)\right| \leqq c(K, r, \delta) e^{-(1-\delta)|x|},
$$

where $D$ represents differentiation with respect to $x_{1}, x_{2}$, and $P_{1}, P_{2}, Q_{1}, Q_{2}$, and $m$ is a multi-index with $|m| \leqq r$.

Proof. We have to examine the limits of Eqs. $(25,32,33)$ as $Z_{1} \rightarrow Z_{2}$. Since the problem is rotationally invariant we can combine this limit with a sequence of rotations and translations, and hence reduce the problem to the case $Z_{1}=x_{1}+i y_{1}=a, Z_{2}=x_{2}+i y_{2}-a$ for $a$ real, so that

$$
\begin{aligned}
& \frac{\partial}{\partial Q}=\frac{-1}{4 a}\left(\partial_{x_{1}}-\partial_{x_{2}}\right)+\frac{i}{4 a}\left(\partial_{y_{1}}-\partial_{y_{2}}\right), \\
& \frac{\partial}{\partial P}=\frac{1}{2}\left(\partial_{x_{1}}+\partial_{x_{2}}\right)-\frac{i}{2}\left(\partial_{y_{1}}+\partial_{y_{2}}\right) .
\end{aligned}
$$

We already know that $\partial_{P}$ gives rise to two square integrable solutions of Eq. (30) from the previous corollary, and these are linearly independent. We next show that $\partial_{Q}$ also gives rise to two such zero modes. Looking at the real term, this amounts to showing that

$$
\lim _{a \rightarrow 0} \frac{\left(n_{1,1}-n_{2,1}\right)}{a}=n_{P_{1}} \in L^{2}
$$

Referring to Eq. (33), this means we are investigating limits of the type:

$$
\begin{aligned}
& \frac{1}{a}\left(\phi_{x_{1}}-\phi_{x_{2}}\right)+\frac{i \phi}{a}\left(\chi_{1,1}-\chi_{2,1}\right) \\
& \quad=\frac{1}{a}\left(u_{x_{1}}-u_{x_{2}}\right) \phi+\frac{i \phi}{a}\left(\Theta_{x_{1}}+\chi_{1,1}-\Theta_{x_{2}}-\chi_{2,1}\right) .
\end{aligned}
$$

To analyse the first term, $w \equiv a^{-1}\left(u_{x_{1}}-u_{x_{2}}\right)$ we notice from Eq. (25) that it is a solution of

$$
-\Delta w+e^{u} w=-\frac{4 \pi}{a}\left(\partial_{x_{1}} \delta_{Z_{1}}-\partial_{x_{2}} \delta_{Z_{2}}\right)
$$


In order to show that $\phi w$ has a well defined square integrable limit we use the regularisation procedure in [JT82]. (See also the proof of Lemma 2.2.) Thus we subtract out the singularities of $w$ by defining

$$
w=-\frac{1}{a}\left(\partial_{x_{1}} \ln \left(1+\frac{\mu}{\left|z-Z_{1}\right|^{2}}\right)-\partial_{x_{2}} \ln \left(1+\frac{\mu}{\left|z-Z_{2}\right|^{2}}\right)\right)+w_{\text {reg }},
$$

where $\mu>8$ is some fixed largish number (see [JT82] or Lemma 2.2). The first two terms give the singular part, and the third is the regular part. We first look at the singular term, which is calculated to be

$$
\frac{-\phi}{a}\left(\frac{2 \mu(x-a)}{|z-a|^{4}+\mu|z-a|^{2}}-\frac{2 \mu(x+a)}{|z+a|^{4}+\mu|z+a|^{2}}\right) .
$$

Now as $a \rightarrow 0$ we can represent $\phi$ as

$$
\phi=(z-a)(z+a) h(z)
$$

with $h$ non-zero (see [JT82]). Thus the singular part has a well defined square integrable limit as $a \rightarrow 0$. For the regular part we find that it satisfies the equation

$$
\begin{aligned}
-\Delta w_{\mathrm{reg}}+e^{u} w_{\mathrm{reg}}= & \frac{4}{a}\left(\frac{\mu}{\left(|z-a|^{2}+\mu\right)^{2}}-\frac{\mu}{\left(|z+a|^{2}+\mu\right)^{2}}\right) \\
& -\frac{e^{u}}{a}\left(\frac{2 \mu(x-a)}{|z-a|^{4}+\mu|z-a|^{2}}-\frac{2 \mu(x+a)}{|z+a|^{4}+\mu|z+a|^{2}}\right) .
\end{aligned}
$$

The right-hand side is uniformly bounded in $L^{2}$ for all values of $a$. From this we obtain the estimate

$$
\int_{\mathbf{R}^{2}}\left|\nabla w_{\text {reg }}\right|^{2}+e^{u} w_{\text {reg }}^{2} \leqq \text { const }
$$

for all values of $a$. This in turn implies that $\left|\Delta w_{\text {reg }}\right|_{L^{2}}$ is bounded independently of $a$. The second term is treated similarly and hence we have proved that $n_{P_{1}}$ extends to a bounded square integrable function as $Z_{1} \rightarrow Z_{2}$. Carrying out the same analysis for derivatives we can see that it is smooth. The imaginary part of $Q$ generates a solution similarly. To see that these solutions are linearly independent, we notice that if this were not the case we would have

$$
\left(u_{x_{1}}-u_{x_{2}}\right)-\left(u_{y_{1}}-u_{y_{2}}\right)=o\left(\left|Z_{1}-Z_{2}\right|\right)
$$

which is easily seen not to be the case.

Finally to complete the proof of the lemma we have to show that the derivatives with respect to $P, Q$ also extend continuously to the point of coincidence. But the analysis of these limits leads to problems of taking limits structurally identical to that just studied. Thus all derivatives

$$
\left(\frac{\partial}{\partial P}\right)^{m}\left(\frac{\partial}{\partial Q}\right)^{n} n_{\mu}
$$

extend continuously to the whole of $M_{2}$, and the lemma is proved. 
This lemma implies that we can write the gauge equivalence classes of solutions to Eqs. $(22-24)$ for $N=2$ as

$$
\begin{aligned}
& a(x ; P, Q) \sim d \chi+a(x ; P, Q), \\
& \phi(x ; P, Q) \sim \phi(x ; P, Q) e^{i \chi},
\end{aligned}
$$

with $P=Z_{1}+Z_{2}, Q=Z_{1} Z_{2}$. We shall see the interesting consequences of this later. Thus for $N=2$ we have written down four linearly independent solutions of the linearised equations which are square integrable. In the general situation we expect there to be $2 N$ square integrable solutions of Eq. (30) which are linearly independent. An informal index calculation of Weinberg justifies this, and we redo his calculation in an appendix in a rigorous fashion.

Theorem 2.6 (Index). The operator D defined in Eq. (30) is a Fredholm operator from $\left(H^{1}\right)^{4}$ to $\left(L^{2}\right)^{4}$ with index $2 N$. Furthermore the adjoint operator is also Fredholm, and $\operatorname{Ker} \mathscr{D}^{*}=\emptyset$.

Notation. We will use $\left\{q_{\mu}\right\}_{1}^{4}$ to denote a generic co-ordinate system on the moduli space, and call $n_{\mu}$ the corresponding solution of Eq. (30) given by Eq. (33) or Lemma 2.5. For $q$ in some small neighbourhood we can choose a specific family of representatives of the equivalence classes of multi-vortices $a(x ; q), \phi(x ; q)$. These can be chosen to depend smoothly on $q$ and such that $a\left(x ; q_{1}\right)-a\left(x ; q_{2}\right)$, $\phi\left(x ; q_{1}\right)-\phi\left(x ; q_{2}\right)$ are square integrable. Of course this latter condition is not satisfied for the representatives given by Eqs. (26) and (27).

The next two results show that the Hamiltonian system defined in the introduction is a smooth function on the cotangent bundle to the moduli space:

Lemma 2.7. The maps

$$
\begin{aligned}
& M_{2} \rightarrow\left(H^{1}\right)^{4} \\
& q_{\mu} \mapsto n_{\mu}
\end{aligned}
$$

are smooth.

Corollary 2.8. The functions $V: M_{2} \rightarrow \mathbf{R}$ and $g: T M_{2} \times T M_{2} \rightarrow \mathbf{R}$ defined by

$$
V(q)=\frac{l}{8} \int_{\mathbf{R}^{2}}\left(1-|\phi(\cdot ; q)|^{2}\right)^{2},
$$

and, for $n_{\mu} \in T_{q} M_{2}$,

$$
g_{q}\left(n_{\mu}, n_{v}\right)=\int_{\mathbf{R}^{2}}\left(n_{\mu}, n_{v}\right)
$$

are smooth.

Proof of Lemma and Corollary. The lemma follows from the smoothness and uniform decay results in Lemmas 2.2 and 2.3. The corollary then follows directly.

2.2. Some Final Comments on the Metric. In [Sam92] the metric is discussed in some detail and we now state some of the results. First of all we notice from Eq. (30) that there is an almost complex structure on the moduli space given by:

$$
\tilde{\alpha} \rightarrow-i \tilde{\alpha} \quad \tilde{\phi} \rightarrow i \tilde{\phi} .
$$


This complex structure is integrable and the metric is in fact Kähler, for any vortex number. This can be seen either by a quotient construction or by the following formula $([\operatorname{Sam} 92])$ :

$$
g=\frac{\pi}{2} \sum\left(\delta_{i j}+2 \frac{\partial \bar{b}_{i}}{\partial z_{j}}\right) d z_{i} d \bar{z}_{j}
$$

where $\frac{\partial \bar{b}_{i}}{\partial z_{j}}=\frac{\partial b_{j}}{\partial \bar{z}_{i}}$ ensures the reality of the kinetic energy. This condition is easily seen to ensure the corresponding Kähler form is indeed closed. The $b_{i}$ coefficients are defined in terms of the local form of $u$ as defined in Eq. (25) - the coefficients of the linear term $z-Z_{i}$ in the expansion of $u$ close to $z=Z_{i}$ is $b_{i}$, and similarly for $\bar{b}_{i}$ after complex conjugation. Another result from [Sam92] we need is an isometric factorisation of the moduli space induced by the action of the translation group. For the two vortex case this allows to write the metric as

$$
g=d Z d \bar{Z}+f d \zeta d \bar{\zeta},
$$

where $Z=Z_{1}+Z_{2}, \zeta=Z_{1}-Z_{2}$. This corresponds to the isometric decomposition

$$
M_{2}=\mathrm{C} \times M_{2}^{0},
$$

where $M_{2}^{0}$ is the relative moduli space, and has metric $g=f d \zeta d \bar{\zeta}$. Writing this in terms of $Q=Z_{1} Z_{2}$, with $Z_{1}+Z_{2}=0$ gives

$$
g=\frac{4 f}{|\zeta|^{2}} d q d \bar{q} \equiv \tilde{f}(|Q|) d Q d \bar{Q}
$$

The theorem above that $P, Q$ form a good co-ordinate system implies the fact that $\tilde{f}$ is finite, i.e. $\frac{f}{|\zeta|^{2}}$ is finite as $\zeta \rightarrow 0$, as asserted in [Sam92]. Finally a remark on the asymptotic behaviour of the metric - we show in the next section (see lemma on asymptotic behaviour) that as the vortices get further apart the solution becomes a superposition of single vortices with an error term which is exponentially small in the vortex separation. This has the consequence (see [Sam92] that the metric asymptotically decouples to $g=\pi\left(d Z_{1}^{2}+d Z_{2}^{2}\right)$, where $\pi$ happens to be the rest mass of a single vortex. Thus the metric on $M_{0}^{2}$ is asymptotically Euclidean. This has a consequence that as long as we use the co-ordinates $Z_{1}, Z_{2}$ for large vortex separations that the metric is uniformly invertible. So we will take the following overlapping co-ordinate patches - choose some number $L$ and for $\left|Z_{1}-Z_{2}\right|<2 L$ we will use $P, Q$ as co-ordinates, and for $\left|Z_{1}-Z_{2}\right|>L$ we have the co-ordinates $Z_{1}, Z_{2}$ themselves. We are then assured that the (Legendre) transformation between $\dot{q}$ and $p$ is uniformly nondegenerate.

\section{The Higgs Mechanism - A Coercive Estimate for the Hessian}

In this section we will use the information from the previous section to derive an estimate for a functional related to the Hessian of the static Abelian Higgs energy equation (16). The basic idea is that, modulo gauge invariance, the Hessian should be equivalent to the $H^{1}$ norm in the subspace orthogonal to the zero modes given in Eq. (33). This is known as the Higgs mechanism in the physics literature. Before 
proving this we notice two apparent difficulties with it:

(i) The Abelian Higgs energy contains only $B=\frac{\partial A_{1}}{\partial x_{2}}-\frac{\partial A_{2}}{\partial x_{1}}$ not the full derivative of $A$. This difficulty disappears when we modify the functional to deal with gauge invariance.

(ii) The potential term which appears is not everywhere positive, thus there are potential problems with bound states. In fact the multi-vortices appear as minima (Eq. (21)) so the only problems are with zero modes (solutions of Eq. (30)), and we are interested in the sub-space orthogonal to these. Thus the relevant question is whether the spectrum extends right down to the origin. Our result could be interpreted as saying the continuous spectrum is pushed away from the origin due to the asymptotic values of the Higgs field when gauge invariance is properly taken care of. We start off by calculating the Hessian of Eq. (16) at a multi-vortex configuration $(a, \phi)$, evaluated on the diagonal $\psi=(\tilde{a}, \tilde{\phi})$ :

$$
\begin{aligned}
\operatorname{Hess}_{a, \phi}(\psi, \psi)= & \frac{1}{2} \int_{\mathbf{R}^{2}}\left\{|d \tilde{a}|^{2}+\sum_{i=1}^{2}\left(\left|D_{i}^{0} \tilde{\phi}\right|^{2}-2 \tilde{a}_{i}\left(i \phi, D_{i}^{0} \tilde{\phi}\right)-2 \tilde{a}_{i}\left(i \tilde{\phi}, D_{i}^{0} \phi\right)\right.\right. \\
& \left.+|\phi|^{2}|\tilde{a}|^{2}+(\phi, \tilde{\phi})^{2}-\frac{1}{2}\left(1-|\phi|^{2}\right)|\tilde{\phi}|^{2}\right\} d^{2} x,
\end{aligned}
$$

where $D_{i}^{(0)}=\partial_{i}-i a_{i}$. We next add on a term to remove the degeneracy which is present as a hangover from gauge invariance. The term is designed to exclude from consideration as zero modes the infinitesimal gauge transformations, so we take the square of the expression Eq. (29). This is because this expression vanishes only orthogonal to the gauge flow. This has been done in the monopole problem also (see [Tau82]). Thus we consider the corrected Hessian,

$$
\begin{aligned}
\overline{\operatorname{Hess}}_{a, \phi}(\psi, \psi)= & \operatorname{Hess}_{a, \phi}(\psi, \psi)+\int_{\mathbf{R}^{2}}(\nabla \cdot \tilde{a}-(i \phi, \tilde{\phi}))^{2} \\
= & \int_{\mathbf{R}^{2}} \sum_{i, j=1}^{2}\left|\nabla_{i} \tilde{a}_{j}\right|^{2}+\sum_{i=1}^{2}\left|D_{i}^{(0)} \tilde{\phi}\right|^{2}+|\phi|^{2}|\tilde{a}|^{2}+|\phi|^{2}|\tilde{\phi}|^{2} \\
& -2 \sum_{i=1}^{2} \tilde{a}_{i}\left(i \tilde{\phi}, D_{i}^{(0)} \phi\right)-\frac{1}{2}\left(1-|\phi|^{2}\right)|\tilde{\phi}|^{2} \\
= & \int_{\mathbf{R}^{2}}\left|\mathscr{D}_{a, \phi} \psi\right|^{2}=(\psi, L \psi)
\end{aligned}
$$

where $\mathscr{D}$ was defined in (30). This expression for $\overline{\text { Hess }}$ has a highly satisfactory form. In fact in answer to the first problem raised above we notice that $\overline{\mathrm{Hess}}_{a, \phi}$ contains all the derivatives of $\tilde{a}$ not just the anti-symmetric one. Secondly, notice that since $|\phi| \rightarrow 1$ exponentially fast as $|x| \rightarrow \infty$ that asymptotically the quadratic form becomes the $H^{1}$ norm at spatial infinity. This is called the Higgs mechanism, and is interpreted as saying that the corresponding particles have mass, and so the fields decay exponentially at infinity. Thinking in terms of spectral theory it leads us to suspect that the essential spectrum is pushed away from the origin, and this is what is behind the next theorem. Notice that we have defined the operator 
$L$ corresponding to the quadratic form $\overline{\mathrm{Hess}}$, and that it is of the form

$$
\begin{gathered}
L=L_{0}+V, \max _{\mathbf{R}^{2}}|V| \leqq c \\
\left(\psi, L_{0} \psi\right)=\int \sum_{i, j=1}^{2}\left|\nabla_{i} \tilde{a}_{j}\right|^{2}+\sum_{i=1}^{2}\left|D_{i}^{(0)} \tilde{\phi}\right|^{2}+|\tilde{a}|^{2}+|\tilde{\phi}|^{2} .
\end{gathered}
$$

Theorem 3.1 (Coercive estimate for Hessian). There exists a number $\gamma$ such that for every $\psi=(\tilde{a}, \tilde{\phi}) \in\left(H^{1, a}\right)^{4}$ which satisfies

$$
(\psi, n)_{L^{2}}=0 \text { for every } n \in \operatorname{Ker} \mathscr{D}
$$

the following coercive estimate holds:

$$
\overline{\operatorname{Hess}}_{a, \phi}(\psi, \psi) \geqq \gamma|\psi|_{1, a}^{2}=\gamma\left(|\nabla \tilde{a}|_{2}^{2}+|\tilde{a}|_{2}^{2}+\left|D^{(0)} \phi\right|_{2}^{2}+|\tilde{\phi}|_{2}^{2}\right)=\gamma\left(\psi, L_{0} \psi\right) .
$$

Remark. It is important that $\gamma$ can be taken independent of the configuration $a, \phi$. Proof. We consider

$$
\tau(q)=\inf _{\psi \in S} \overline{\operatorname{Hess}}_{a, \phi}(\psi, \psi), \quad S=\left\{\psi:|\psi|_{H^{1}}=1,(\psi, n)_{2}=0 \text { for all } n \in \operatorname{Ker} \mathscr{D}\right\} .
$$

We shall write $\tau(q)$ to indicate dependence on the configuration as parametrised by $q$ in the course of showing that such dependence can be removed, i.e. that we can find a number $0<\gamma \leqq \tau(q) \forall q$.

To achieve the first part we fix $a, \phi$, and assume that $\tau(a, \phi)=0$, then by density we have a $C_{0}^{\infty}$ sequence $\psi^{i}$ such that

$$
\psi^{i} \rightarrow \psi \in H^{1}, \quad \overline{\operatorname{Hess}}_{a, \phi}\left(\psi^{i}, \psi^{i}\right) \rightarrow 0, \quad\left|\psi^{(i)}\right|_{1, a}=1, \quad\left(\psi^{(i)}, n_{\mu}\right)=0,
$$

and we will try to draw a contradiction. Integrating by parts we get

$$
\overline{\operatorname{Hess}}_{a, \phi}\left(\psi^{i}, \psi^{i}\right)=\left|\mathscr{D} \psi^{i}\right|_{2}^{2} \rightarrow 0,
$$

so in the limit we have $|\mathscr{D} \psi|=0$. But since $\psi^{i} \rightarrow \psi$ in $H^{1}$, it follows that

$$
(\psi, n)_{2}=\lim \left(\psi^{i}, n\right)=0
$$

so that $\psi=0$. To draw a contradiction we use the fact that by the Rellich lemma there is strong $L^{2}$ convergence on compact sets. We apply this to the formula:

$$
\begin{aligned}
\left|\psi^{i}\right|_{H^{1, a}}= & \overline{\operatorname{Hess}}_{a, \phi}\left(\psi^{i}, \psi^{i}\right)-\int_{\mathbf{R}^{2}} \frac{3}{2}\left(1-|\phi|^{2}\right)\left|\tilde{\phi}^{i}\right|^{2}+\left(1-|\phi|^{2}\right)\left|\tilde{a}^{i}\right|^{2} \\
& +4\left(\tilde{\phi},-i a^{i} \cdot D \phi\right) \\
= & \overline{\operatorname{Hess}}_{a, \phi}\left(\psi^{i}, \psi^{i}\right)+\int_{\mathbf{R}^{2}} Q .
\end{aligned}
$$

Now given any $\varepsilon$ we can choose a radius $r(\varepsilon)$ such that

$$
\left(1-|\phi|^{2}\right),\left|D^{(0)} \phi\right|<\varepsilon \text { for }|x|>r(\varepsilon) \text {. }
$$

Thus calling the ball of radius $r(\varepsilon), B_{r(\varepsilon)}$ we find that as $i \rightarrow \infty$ :

$$
\int_{B_{r(e)}} Q \rightarrow 0 \text { by Rellich's lemma }
$$


for every $\varepsilon$. But also we see that since $\left|\psi^{i}\right|_{1, a}=1$ we have the following estimate uniform in $i$ :

$$
\int_{\mathbf{R}^{2}-B_{r(\varepsilon)}} Q=O(\varepsilon)
$$

This leads to a contradiction since $\left|\psi^{i}\right|=1$ and $\overline{\operatorname{Hess}}_{a, \phi}\left(\psi^{i}, \psi^{i}\right) \rightarrow 0$. Thus we deduce that for each $q, \tau(q)>0$. The proof of the theorem will be completed by the following three lemmas which remove the dependence on the background configuration.

Lemma 3.2 (Continuous dependence). The dependence of $\tau$ on the parameters $q$ of the moduli space is continuous.

Lemma 3.3 (Single Vortex). For a single vortex, $\tau$ is independent of $q$ (by translation invariance).

The next lemma states that outside compact regions of the moduli space $\tau$ is bounded below uniformly.

Lemma 3.4 (Asymptotic Lemma). Consider any sequence of vortex configurations $q_{n} \in M_{2}$ in which the separation of the two vortices becomes infinite $\left|Z_{1}-Z_{2}\right| \rightarrow \infty$. Then there is a fixed positive number such that $\tau\left(q^{i}\right) \geqq \alpha$ for all such sequences.

The middle lemma requires no proof. The other two are now proved in turn.

Proof of Continuity Lemma. Here we can either apply the general results of chapter five of [Kat66] or use a direct argument as follows. Let $q_{n} \rightarrow q$ be a sequence of points on the moduli space, and write the corresponding configurations $a\left(\cdot ; q_{n}\right)$, $\phi\left(\cdot ; q_{n}\right)$ as $c\left(q_{n}\right)$, then for each $n$ we have $\tau_{n}=\tau\left(q_{n}\right)$ and our task is to show that $\tau_{n} \rightarrow \tau=\tau(q)$. We need the following two results to prove this.

Claim $A$ : If we write $P_{n}$ for the projection operator onto the $L^{2}$-orthogonal complement of $\operatorname{Ker} \mathscr{D}_{c\left(q_{n}\right)}$ then $P_{n} \rightarrow P$, in the strong operator topology induced from $H^{1, a}$, where $P$ is the corresponding projection operator for the limiting configuration $q$ and $a$ is the connection corresponding to $q$. This follows almost immediately from Lemma 2.3 and its corollary if we notice that $\left(P_{n}-P\right) \psi=\sum\left(\psi, n_{v}\right) n_{v}-\sum\left(\psi, n_{v}^{q_{n}}\right) v_{v}^{q_{n}}$, where $n_{v}^{q_{n}}$ is a basis for $\operatorname{Ker} \mathscr{D}_{c\left(q_{n}\right)}$.

Claim B: There exists a positive increasing function $\delta$, such that $\delta(r) \rightarrow 0$ as $r \rightarrow 0$, with the property that

$$
\overline{\operatorname{Hess}}_{c(q)}(\psi, \psi)-\overline{\operatorname{Hess}}_{c\left(q^{\prime}\right)}(\psi, \psi) \leqq \delta\left(\left|q-q^{\prime}\right|\right)|\psi|_{L^{2}} .
$$

To see this we just look at the expression for the Hessian and notice that the coefficients are continuous functions of $q$ in the uniform topology.

We will now show that $\tau \leqq \lim \inf \tau_{n}$, and $\tau \geqq \lim \sup \tau_{n}$. To see the former imagine that $\tau(q)>\lim \inf \tau\left(q_{n}\right)-$ then we have minimising sequences of unit $L^{2}$ norm such that

$$
\overline{\operatorname{Hess}}_{c\left(q_{n}\right)}\left(\psi_{n}^{i}, \psi_{n}^{i}\right) \rightarrow \tau\left(q_{n}\right) .
$$

Now project $\psi_{n}^{i}$ onto the subspace orthogonal to Ker $\mathscr{D}_{(c(q))}$ - this produces a new sequence $\tilde{\psi}_{n}^{i}$, with the property that $\left|\tilde{\psi}_{n}^{i}-\psi_{n}^{i}\right|_{H^{1}} \leqq \varepsilon_{n}$, where $\varepsilon_{n} \rightarrow 0$ as $n \rightarrow \infty$, and is independent of $i$, from Claim A. Next we use Claim B to deduce that

$$
\overline{\operatorname{Hess}}_{c\left(q_{n}\right)}\left(\tilde{\psi}_{n}^{i}, \tilde{\psi}_{n}^{i}\right)=\left|\mathscr{D}_{c(q)} \tilde{\psi}_{n}^{i}\right|^{2}=\left|\mathscr{D}_{c\left(q_{n}\right)} \psi_{n}^{i}\right|^{2}+\tilde{\varepsilon}_{n}=\overline{\operatorname{Hess}}_{c\left(q_{n}\right)}\left(\psi_{n}^{i}, \psi_{n}^{i}\right)+\tilde{\varepsilon}_{n},
$$


where $\tilde{\varepsilon}_{n} \rightarrow 0$ as $n \rightarrow \infty$ and is independent of $i$. From this we can draw the conclusion $\tau \leqq \liminf \tau_{n}$ by using Cantor diagonalisation to produce a subsequence which contradicts the assumption. An almost identical argument yields the fact that $\tau \geqq \lim \sup \tau_{n}$ - we assume that $\tau<\lim \sup \tau_{n}$, and use a minimising sequence for the limiting configuration $q$ to produce "almost minimising" sequences for nearby configurations $q_{n}$. The "almost minimising" sequences give lower values for $\tau_{n}$ than possible if the assumption were true.

Proof of Asymptotic Lemma. This hinges on the fact that when the two vortices are far apart the spectrum should be as for a single vortex. Thus we will show that if $\tau(q)$ approached zero at infinity of the moduli space then $\tau$ for a single vortex would be zero contrary to the lemma above. We first of all notice that by translation invariance it only depends on $Z_{1}-Z_{2}$. Furthermore by translation and rotation invariance we can take $Z_{1}=d, Z_{2}=-d$, with $d$ real. We will write $u_{0}$ for the solution of the single vortex problem

$$
-\Delta u_{0}+e^{u_{0}}-1=-4 \pi \delta_{0}
$$

then the solution of

$$
-\Delta u+e^{u}-1=-4 \pi\left(\delta_{d}+\delta_{d}\right)
$$

can be written as

$$
u=u_{0}(z-d)+u_{0}(z+d)+\tilde{u},
$$

where

$$
-\Delta \tilde{u}+e^{u_{0}(z-d)+u_{0}(z-d)}\left(e^{\tilde{u}}-1\right)=-\left(e^{u_{0}(z-d)}-1\right)\left(e^{u_{0}(z+d)}-1\right) .
$$

We want to show $\tilde{u}$ is small for large $d$ :

Claim. There exists constants $C, a$ such that

$$
|\tilde{u}|_{\infty},|\tilde{u}|_{H^{2}},\left|\frac{\partial \tilde{u}}{\partial q}\right|_{\infty},\left|\frac{\partial \tilde{u}}{\partial q}\right|_{H^{2}} \leqq C e^{-a d} .
$$

This is proved after we show that it allows us to prove the asymptotic lemma. We divide the plane into three regions

$$
\mathbf{R}^{2}=B_{1} \cup B_{2} \cup\left(\mathbf{R}^{2}-B_{1}-B_{2}\right)=B_{1} \cup B_{2} \cup \mathscr{R},
$$

where $B_{1}, B_{2}$ are balls of large radius $r$ around $d,-d$ respectively. Now for $d \gg 1$ we see that $u$ is the sum of three exponentially small terms outside the two balls. Applying this to Eq. (47) we find that

$$
|\mathscr{D} \psi|_{L^{2}(\mathscr{R})}=|\psi|_{H^{1} \mathscr{R}}+o(1) .
$$

Now consider a sequence of vortex configurations with $d=d_{i} \rightarrow \infty$, where by assumption we can find a sequence $\psi^{i}$ such that

$$
\left|\psi^{i}\right|_{H^{1}}=1, \quad\left|\mathscr{D}_{d^{i}} \psi^{i}\right|_{2} \rightarrow 0
$$

where $d_{i}$ refers to the background two vortex configuration corresponding to vortices centred at $d_{i},-d_{i}$. Then we deduce from the previous equation that $\left|\psi^{i}\right|_{H^{1}(\mathscr{R})}$ becomes arbitrarily small, and so one of $\left|\psi^{i}\right|_{H^{1}\left(B_{1}\right)},\left|\psi^{i}\right|_{H^{1}\left(B_{2}\right)}$ must be bigger than, say $1 / 3$. We can now see our contradiction - as $d \rightarrow \infty$ if we restrict to 
one of the balls we have a sequence with

$$
\left|\mathscr{D}_{d_{0}} \psi^{i}\right| \rightarrow 0, \quad\left|\psi^{i}\right|>\frac{1}{3},
$$

where $d_{0}$ is a background one-vortex configuration. But this would imply that $\gamma=0$ for a single vortex, which is not the case by translation invariance as expressed in the lemma above.

Proof of Claim. First of all notice that by the maximum principle $\tilde{u}<0$. Next multiply by $\left(e^{\tilde{u}}-1\right)$ to obtain:

$$
\int e^{\tilde{u}}|\nabla \tilde{u}|^{2}+e^{u_{0}(z-d)+u_{0}(z-d)}\left(e^{\tilde{u}}-1\right)^{2}=\int\left(e^{u_{0}(z-a)}-1\right)\left(e^{u_{0}(z+a)}-1\right)\left(e^{\tilde{u}}-1\right) .
$$

Now from the negativity of $\tilde{u}$, and the fact that $e^{u_{0}}-1$ decays exponentially, we find

$$
\int e^{\tilde{u}}|\nabla \tilde{u}|^{2}+e^{u_{0}(z-d)+u_{0}(z-d)}\left(e^{\tilde{u}}-1\right)^{2}=O\left(e^{-a d}\right) .
$$

Substituting this into the equation we can estimate $\Delta \tilde{u}$ to be exponentially small also which proves the proposition.

Theorem 3.1 is the key to our method, which will be based on the fact that the Hessian is "almost conserved" as we shall see in Sect. 5. We will need the following corollary.

Corollary 3.5. Consider the quadratic form defined by

$$
Q(\psi, \psi)=\overline{\operatorname{Hess}}_{a, \phi}(\psi, \psi)+\overline{\operatorname{Hess}}_{a, \phi}(L \psi, L \psi) .
$$

Then on the subspace of $H^{3, a}$ defined by the conditions

$$
\left(\psi, n_{\mu}\right)_{L^{2}}=0 \text { for every } n_{\mu} \in \operatorname{Ker} \mathscr{D}
$$

there exists a number $c$ such that

$$
c^{-1}|\psi|_{3, a} \leqq Q(\psi, \psi) \leqq c|\psi|_{3, a} .
$$

Proof. We will use $c$ for a variable constant. We already know that on this subspace

$$
c^{-1}|\psi|_{1, a} \leqq \overline{\operatorname{Hess}}_{a, \phi}(\psi, \psi) \leqq c|\psi|_{1, a}
$$

and we know that $\left(L \psi, n_{\mu}\right)=0$ since $L n_{\mu}=0$. From this we see that

$$
\begin{aligned}
|\psi|_{1, a}+|L \psi|_{1, a} & \leqq \overline{\operatorname{Hess}}_{a, \phi}(\psi, \psi)+\overline{\operatorname{Hess}}_{a, \phi}(L \psi, L \psi) \\
& \leqq c\left(|L \psi|_{1, a}+|\psi|_{1, a}\right) \leqq c|\psi|_{3, a} .
\end{aligned}
$$

Now looking at the expression in $(48,49)$ for $L$ we see that

Claim. There exists a number $c$ such that

$$
c^{-1}|\psi|_{2, a} \leqq|\psi|_{L^{2}}+|L \psi|_{L^{2}} \leqq c|\psi|_{2, a} .
$$

To see this notice that from the definition of $L_{0}$,

$$
\begin{aligned}
|\psi|_{2, a} & \leqq c\left(|\psi|_{L^{2}}+|\Delta \psi|_{L^{2}}\right) \\
& \leqq c\left(|\psi|_{L^{2}}+\left|L_{0} \psi\right|_{L^{2}}\right) \\
& \leqq c\left(|\psi|_{L^{2}}+|L \psi|_{L^{2}}+|V \psi|_{L^{2}}\right) \\
& \leqq c\left(|\psi|_{L^{2}}+|L \psi|_{L^{2}}\right) .
\end{aligned}
$$


Now we see that

$$
\begin{aligned}
|\psi|_{3, a} & \leqq|\psi|_{2, a}+|\nabla \psi|_{2, a} \\
& \leqq c\left(|\psi|_{L^{2}}+|L \psi|_{L^{2}}+|\nabla \psi|+|L \nabla \psi|_{L^{2}}\right) \\
& \leqq c\left(|\psi|_{L^{2}}+|L \psi|_{L^{2}}+|\nabla L \psi|_{L^{2}}\right) \\
& \leqq c\left(|\psi|_{L^{2}}+|L \psi|_{1, a}\right) \leqq Q(\psi, \psi),
\end{aligned}
$$

where the final equation follows from Eq. (51). This proves the corollary.

\section{The Ansatz}

In this section we will write down an ansatz for the solution to describe the slow (low-energy) dynamics of vortices. Very roughly this consists of writing the solution as

$$
A=a(\cdot ; q(t))+\varepsilon^{2} \tilde{a}, \quad \Phi=\phi(\cdot ; q(t))+\varepsilon^{2} \tilde{\phi} .
$$

We then apply a Fredholm type condition to obtain equations for the evolution of the parameters $q_{\mu}$. This condition, Eq. (13), was discussed in the remarks following the statement of the main theorem, and should be thought of as choosing $q$ as a function of time in order to minimise the $L^{2}$ distance of the solution from the (modulating) multi-vortex solution. The reader interested in a more leisurely description of the asymptotic situation may refer to the companion paper [Stu].

Of course the real justification for choosing the time evolution $q(t)$ so as to satisfy (13) is that this leads to a rigorous estimate of the error terms $(\tilde{a}, \tilde{\phi})$, as shown in the next section. Nevertheless we will give an informal "derivation" of those conditions here for motivation. Our ultimate aim is to estimate the error terms using the Hessian, which is a conserved quantity for the equations linearised about a static multi-vortex. We will see later that it is approximately conserved by the full equations for $\tilde{a}, \tilde{\phi}$ on a slowly modulating background. The difficulty with using the Hessian however is that it is not a coercive quadratic form as it stands. We saw in Sect. 3 that in order to make it coercive we have to restrict the subspace on which it is evaluated in two ways:

(i) Ensure that $\psi=\tilde{a}, \tilde{\phi}$ satisfies the gauge orthogonality condition:

$$
\nabla \cdot \tilde{a}-(i \varphi, \tilde{\phi})=0 \text {. }
$$

To see that this is possible, notice that if $A=a(\cdot ; q(t))+\varepsilon^{2} \tilde{a}, \Phi=\phi(\cdot ; q(t))+\varepsilon^{2} \tilde{\phi}$ is a solution for which this is not satisfied, then a gauge transformation

$$
A \rightarrow A+\varepsilon^{2} d \xi, \quad \Phi \rightarrow \Phi e^{i \varepsilon^{2} \xi},
$$

with

$$
\Delta \xi=(i \phi, \tilde{\phi})-\operatorname{div} \tilde{a}+\varepsilon^{-2}|\phi|^{2} \sin \varepsilon^{2} \xi
$$

will produce a solution satisfying the condition.

(ii) Ensure that $\psi=\tilde{a}, \tilde{\phi}$ is orthogonal to the zero modes $n_{\mu}$, which are the zero eigenvalues of the Hessian. This is condition (13). To motivate this we expand upon the comments in the introduction. We are trying to match our solution to a multi-vortex with parameters $q(t)$, so we try to minimise the $L^{2}$ distance, i.e. we 
determine $q(t)$ by the minimisation problem

$$
\min _{q(t) \in M_{2}} \int_{\mathbf{R}^{2}}|A(t, \cdot)-a(\cdot ; q(t))|^{2}+\Phi(t, \cdot)-\left.\phi(\cdot ; q(t))\right|^{2} .
$$

We now show that if $\tilde{\phi}$ are smooth, compactly supported and satisfy the gauge orthogonality condition, that this minimisation leads to condition (13). The differential conditions for the minimum are

$$
\int\left(\tilde{a}, \frac{\partial a}{\partial q_{\mu}}\right)+\left(\tilde{\phi}, \frac{\partial \phi}{\partial q_{\mu}}\right)=0
$$

which makes sense on account of our assumptions on $\tilde{a} \tilde{\phi}$. We now take advantage of the gauge orthogonality condition to add on the infinitesimal gauge transformation given by Lemma 2.3. This converts the partial derivatives with respect to $q_{\mu}$ into the zero modes $n_{\mu}$, and hence to the condition

$$
\int\left((\tilde{a}, \tilde{\phi}), n_{\mu}\right)=0 \text {. }
$$

But this is condition (13) and makes sense for any $\tilde{a}, \tilde{\phi}$ square integrable, and so will be adopted as the condition to choose $q(t)$.

We now write down the ansatz properly and apply condition (13). The starting point is the time dependent equations corresponding to the energy (Eq. (16)):

$$
\begin{gathered}
-\Delta A_{0}+\partial_{t}\left(\partial_{i} A_{i}\right)=J_{0}, \\
\partial_{t}^{2} A_{i}-\Delta A_{i}-\partial_{t} \partial_{i} A_{0}+\partial_{i}\left(\partial_{j} A_{j}\right)=J_{i}, \\
D_{0}^{2} \Phi-D_{1}^{2} \Phi-D_{2}^{2} \Phi=\frac{\lambda}{2} \Phi\left(1-|\Phi|^{2}\right),
\end{gathered}
$$

where $J_{\mu}=\left(i \Phi, D_{\mu} \Phi\right)$. In order to write down the ansatz it is convenient to introduce a slow time variable $\tau=\varepsilon t$. The only feature of the rough ansatz stated above that is not quite right is that we must allow for the possibility that at time $t$ the solution looks like a gauge transformed multi-vortex. Thus we introduce a time dependent base gauge change $\Xi$. This leads to the ansatz:

$$
\begin{aligned}
\Phi(t, x) & =\left[\phi(x ; q) e^{i \Xi}+\varepsilon^{2} \tilde{\phi}(t, x)\right]=\varphi(x ; q)+\varepsilon^{2} \tilde{\phi}, \\
A_{0} & =\varepsilon^{3} \tilde{\tilde{a}}_{0}, \\
A_{i} & =a_{i}(x ; q)+\partial_{i} \Xi+\varepsilon^{2} \tilde{a}_{i}(t, x)=\alpha(x ; q)+\varepsilon^{2} \tilde{a}_{i},
\end{aligned}
$$

where we have defined $\varphi=\phi e^{i \Xi}$, and $q_{\mu}$ is shorthand for the two possible parametrisations of the two vortex solutions discussed in Sect. 2.1: $Z_{1}, Z_{2}$, or $P, Q$. We require $\tilde{a}, \tilde{\phi}$ to satisfy the gauge orthogonality condition and condition (13). Finally the base gauge change $\Xi$ of the multi-vortex is a slowly varying function chosen such that

$$
\left(\frac { d } { d t } \left(a_{i}\left(x ; q_{\mu}\right)+\partial_{i} \Xi, \frac{d}{d t}\left(\phi\left(x ; q_{\mu} e^{i \Xi}\right)\right)=\left(\frac{d \alpha}{d t}, \frac{d \varphi}{d t}\right)\right.\right.
$$

satisfies the gauge orthogonality condition Eq. (29). It is these two conditions which allow $A_{0}=O\left(\varepsilon^{3}\right)$. The reader will see by referring to the proof of Lemma 2.3 that this choice of base gauge ensures that

$$
\left(\frac{d \alpha}{d \tau}, \frac{d \varphi}{d \tau}\right)=\sum_{\mu=1}^{4} \dot{q}_{\mu} n_{\mu}
$$


which we shall use later. We also comment that $\Xi(t)$ will solve the equation

$$
-\Delta \Xi_{t}+|\phi|^{2} \Xi_{t}=-|\phi|^{2} \Theta_{t}
$$

so that $t \rightarrow \Xi \in C^{\infty}\left(\mathbf{R}^{2}\right)$ will be twice differentiable for $q \in C^{2}$. Substitution of the ansatz leads to the following equations for the error terms:

$$
\begin{gathered}
-\Delta \tilde{\tilde{a}}_{0}+|\phi|^{2} \tilde{\tilde{a}}_{0}=-2\left(i \tilde{\phi}, \varphi_{\tau}\right)+\varepsilon j_{0}, \\
\partial_{t}^{2} \tilde{a}_{i}-\Delta \tilde{a}_{i}+|\phi|^{2} \tilde{a}_{i}-2\left(i \tilde{\phi}, D_{i}^{(0)} \phi\right)=-\partial_{\tau}^{2}\left(a_{i}+\partial_{i} \Xi\right)+\varepsilon j_{1}, \\
\partial_{t}^{2} \tilde{\phi}-\sum_{i=0}^{2}\left(D_{i}^{(0)}\right)^{2} \tilde{\phi}-2 i \tilde{a}_{i} D_{i}^{(0)} \varphi+|\phi|^{2} \tilde{\phi}-\frac{1}{2}\left(1-|\phi|^{2}\right) \tilde{\phi} \\
=-\partial_{\tau}^{2} \varphi+\frac{l}{2} \varphi\left(1-|\phi|^{2}\right)+\varepsilon j_{3},
\end{gathered}
$$

where we use the notation $D_{i}^{(0)} \phi=\partial_{i} \phi-i a_{i} \phi$, and the error terms are in the first appendix. It is convenient to rewrite the last two equations in terms of $\psi=(\tilde{a}, \tilde{\phi})$ as

$$
\psi_{t t}+L \psi=k+\varepsilon j,
$$

where $L=L(t)$ was defined in Sect. 3 by $(\psi, L \psi)=\overline{\operatorname{Hess}}_{\alpha, \varphi}(\psi, \psi)$ and

$$
k=\left(-\partial_{\tau}^{2}\left(a_{i}+\partial_{i} \Xi\right),-\partial_{\tau}^{2} \varphi+\frac{l}{2} \varphi\left(1-|\phi|^{2}\right)\right)
$$

and $j$ represents the non-linear terms, which are given in the appendix. The next step is to apply the condition in Eq. (13) to derive equations for the evolution of $q(t)$, and then to show that these equations ensure that Eqs. (61-63) will have bounded solutions for large times.

4.1. Derivation of the Modulation Equations. The modulation equations for the parameters $q_{\mu}$ are deduced from the additional condition that $(\tilde{a}, \tilde{\phi})$ be orthogonal to the zero modes Eq. (33), or Lemma 2.5 (depending on the co-ordinate system), and so we require

$$
\left((\tilde{a}, \tilde{\phi})^{t}, n_{\mu}\right)=0
$$

which implies, using the slow time variable $\tau=\varepsilon t$,

$$
\left(\left(\tilde{a}_{t t}, \tilde{\phi}_{t t}\right)^{t}, n_{\mu}\right)=-\varepsilon^{2}\left((\tilde{a}, \tilde{\phi})^{t}, \frac{\partial^{2} n_{\mu}}{\partial \tau^{2}}\right)-2 \varepsilon\left(\left(\tilde{a}_{t}, \tilde{\phi}_{t}\right)^{t}, \frac{\partial n_{\mu}}{\partial \tau}\right) .
$$

We apply this, using the fact that $n_{\mu}$ solves the linearised Eq. (30), which implies $L n_{\mu}=0$, to obtain the modulation equations:

where

$$
\left(\left(\partial_{\tau}^{2}\left(\alpha_{i}\right), \partial_{\tau}^{2} \varphi\right), n_{\mu}\right)-\frac{l}{2}\left(\varphi\left(1-|\phi|^{2}\right), n_{\mu}\right)=\varepsilon h,
$$

$$
h=\left(\left(j_{1}, j_{2}, j_{3}\right), n_{\mu}\right)+\varepsilon\left(\psi, \partial_{\tau}^{2} n_{\mu}\right)+2\left(\psi_{t}, \partial_{\tau} n_{\mu}\right) .
$$

These equations are rather unwieldy as they stand, so we will first give a geometric interpretation of the first term (the inertial term), and then prove that they have solutions which are bounded for long times thus justifying the asymptotics. We shall then see about drawing phenomenological consequences. 
4.2. Interpretation of the Inertial Terms. In this section we show that our asymptotics are consistent with the Abelian Higgs model version of Manton's hypothesis ([Man82]), which we described in the introduction. This means we consider the scattering of vortices in the Bogomolny limit, i.e. with $l=0$, which is to highest described by the equation

$$
\left(\left(\partial_{\tau}^{2}(\alpha), \partial_{\tau}^{2} \varphi\right), n_{\mu}\right)=0
$$

We shall interpret this equation as the equation for geodesics on the moduli space. The metric on the moduli space is the $L^{2}$ inner product of the gauge orthogonal solutions to the linearised equations given in Eq. (33) and Lemma 2.5. The geodesics $\tau \rightarrow q(\tau)$ are extrema of the integral

$$
\int\left(n_{\mu}, n_{\lambda}\right) \dot{q}_{\mu} \dot{q}_{\nu} d t
$$

where the notation $q_{\mu}$ refers to either of the co-ordinate systems for the two vortex moduli space, $Z_{1}, Z_{2}$ or $p, q$. We choose some representatives of the equivalence classes of multi-vortices as discussed in Sect. 2.1 and $n_{\mu}$ are the corresponding solutions of Eq. (30). The Euler Lagrange equation is

$$
-\frac{d}{d \tau}\left(\left(n_{\lambda}, n_{\kappa}\right) \dot{q}_{\kappa}\right)+\frac{1}{2}\left(\frac{\partial n_{\mu}}{\partial q_{\lambda}}, n_{\kappa}\right) \dot{q}_{\mu} \dot{q}_{\kappa}+\frac{1}{2}\left(\frac{\partial n_{\kappa}}{\partial q_{\lambda}}, n_{\mu}\right) \dot{q}_{\mu} \dot{q}_{\kappa}=0 .
$$

To see that this corresponds to the inertial terms in the modulation equation we need the following identity.

Claim.

$$
-\left(\frac{d n_{\lambda}}{d \tau}, n_{\kappa}\right) \dot{q}_{\kappa}+\frac{1}{2}\left(\frac{\partial n_{\mu}}{\partial q_{\lambda}}, n_{\kappa}\right) \dot{q}_{\mu} \dot{q}_{\kappa}+\frac{1}{2}\left(\frac{\partial n_{\kappa}}{\partial q_{\lambda}}, n_{\mu}\right) \dot{q}_{\mu} \dot{q}_{\kappa}=0 .
$$

Proof. To see this we notice that this will follow if $Q=\left(n_{\lambda, q_{\mu}}-n_{\mu, q_{\lambda}}, n_{\kappa}\right)=0$. To see why this is zero it is convenient to introduce the covariant derivative on the moduli space defined by $D_{\mu} \equiv P_{\alpha, \varphi} \frac{\partial}{\partial q_{\mu}}$, where $P_{\alpha, \varphi}$ is the projection in $L^{2}$ onto $T_{\alpha, \varphi}$, thus $\left(1-P_{\alpha, \varphi}\right)$ is the projection orthogonal to $T_{\alpha, \varphi}$, and so $Q=\left(D_{\mu} n_{\lambda}-D_{\lambda} n_{\mu}, n_{\kappa}\right)$ since $n_{\kappa} \in T_{\alpha, \varphi}$. We now use the fact that the $n_{\mu}$ have the structure given in Eq. (33), which we write schematically as

$$
n_{\mu}=\frac{\partial}{\partial q_{\mu}}(A)+g, \quad\left(g, n_{\mu}\right)=0,
$$

where $g$ is an infinitesimal gauge transformation, and the latter relation follows from the fact that $n_{\mu}$ satisfies the gauge orthogonality requirement. We see immediately that the contribution to $Q$ from the first term $\left(\partial_{q_{\mu}} A\right)$ vanishes. To see that the contribution from the second term also vanishes we first proceed formally. Recall that by definition of $T_{\alpha, \varphi}$ we have $\left(g, n_{\kappa}\right)=0$ so differentiating this we find, at least formally, $\left(D_{\mu} g, n_{\kappa}\right)=-\left(g, D_{\mu} n_{\kappa}\right)$. But $\left(g, D_{\mu} n_{\kappa}\right)$ since $D_{\mu} n_{\kappa} \in T_{\alpha, \varphi}$ by definition of $D_{\mu}$, and so satisfies the gauge orthogonality condition. To complete the proof of the claim we comment that, even though $g \notin L^{2}$, since the $n_{\mu}$ are exponentially decaying in space and smooth all these formal calculations are justified. 
Combining these two equations we are led to the following equations for geodesics:

$$
-\left(n_{\lambda}, n_{\kappa}\right) \ddot{q}_{\kappa}-\left(n_{\lambda}, \frac{d n_{\kappa}}{d \tau}\right) \dot{q}_{\kappa}=0 .
$$

We can now see by differentiating (60) that this is equivalent to Eq. (68), Thus we see that the asymptotic equation takes the form of an inertial term "mass $\times$ acceleration" in the form of geodesics, and a force term $-\frac{l}{2}\left(\varphi\left(1-|\phi|^{2}\right), n_{\mu}\right)$ due to the departure from the Bogomolny regime $\left(\lambda=1 \pm \imath \varepsilon^{2}\right)$. We now show that to highest order the modulation equations are in Hamiltonian form, with Hamiltonian $H: T^{*} M_{2} \rightarrow \mathbf{R}:$

$$
H=\frac{1}{2} g(p, p)+V(q),
$$

where $p$ is the momentum which is determined from $\dot{q}$ from the Legendre transformation $p_{\mu}=\sum_{\kappa}\left(n_{\mu}, n_{\kappa}\right) \dot{q}_{\kappa}, g$ is the dual metric defined by $g(p, p)=$ $\sum_{\mu, v}\left(n_{\mu}, n_{v}\right) \dot{q}_{\mu} \dot{q}_{v}$, and $V: M_{2} \rightarrow \mathbf{R}$ is a potential energy defined on the moduli space by

$$
V(q)=\frac{l}{8} \int_{\mathbf{R}^{2}}\left(1-|\phi|^{2}\right)^{2} .
$$

The transformation $\dot{q} \rightarrow p$ is uniformly non-degenerate on account of the comments in Sect. 2.2, and the function $V: M_{2} \rightarrow \mathbf{R}$ is smooth. The Hamilton equations are

$$
\frac{d p_{0, \mu}}{d \tau}=-\frac{\partial H}{\partial q_{0, \mu}} \quad \frac{d q_{0, \mu}}{d \tau}=\frac{\partial H}{\partial p_{0, \mu}}
$$

of which the first corresponds to the modulation equation and the second to the definition of $p$.

We now turn to proving that the asymptotics provides good approximations to the true solutions for small $\varepsilon$.

\section{Proof of Validity of Approximation}

In this section we will prove that the expansions suggested by the formal asymptotics of the previous section provide good approximations to the solutions of the initial value problem for Eqs. (54-56), with initial data of the form

$$
\begin{aligned}
A(0, x) & =a(x ; q(0))+\varepsilon^{2} \tilde{a}(0), & A_{t}(0) & =\varepsilon \sum_{\mu} \dot{q}_{\mu}(0) n_{\mu}^{1}+\varepsilon^{2} \tilde{a}_{t}(0), \\
\Phi(0, x) & =\phi(x ; q(0))+\varepsilon^{2} \tilde{\phi}(0), & \Phi_{t}(0, x) & =\varepsilon \sum \dot{q}_{\mu}(0) n_{\mu}^{2}+\varepsilon^{2} \tilde{\phi}_{t}(0) .
\end{aligned}
$$

Notice that our asymptotics have led us to study a system composed of elliptic and hyperbolic differential equations coupled to ordinary differential equations - see Eqs. (61-66). The strategy is first of all to prove a local existence theorem for this system in a suitable space. We then use an a priori estimate to show that the corresponding norm is bounded for a time of $O\left(\frac{1}{\varepsilon}\right)$, and thus continue the 
solution for such times of $O\left(\frac{1}{\varepsilon}\right)$ by applying the local existence theorem repeatedly. The relevant norm is determined by the fact that for the linearised evolution (i.e. that formally obtained from (61-63) by putting $\varepsilon=0$ ) the Hessian of the energy (16) is almost conserved. We proved in Sect. 3 however that as long as $\tilde{a}, \tilde{\phi}$ satisfies the orthogonality conditions in Eqs. (12) and (13) then Hess $(\psi)$ is equivalent to the $H^{1}$ norm of $\psi$. This suggests that we should try to use $H^{1}$ for our norm. However we can do better than this by noticing that if we consider $L \psi$ then this will satisfy the condition (13) (on account of the fact that $L$ is self adjoint and $L n_{\mu}=0$ ). And in addition we see that to highest order it satisfies an equation of the form

$$
(L \psi)_{t t}+L(L \psi)=L \kappa+O(\varepsilon)
$$

and so Hess $(L \psi)$ is also approximately conserved. This will give us information on $|\psi|_{3}$ and hence pointwise $\left(L^{\infty}\right)$ control, by Corollary 3.5.

Notation. When we write $L$ it is to be understood that this means the operator defined in Sect. 3 with background configuration $\alpha(\cdot ; q(t)), \varphi(\cdot ; q(t))$. This will be suppressed for simplicity. The error terms in the equations are given in an appendix. We will use $c$ for variable constants, and $c(\cdot, \ldots, \cdot)$ for positive increasing functions depending on some number of variables used in estimation. Also $\dot{q}, \ddot{q}, q^{(3)}$ denote $\frac{d q}{d \tau}=\frac{1}{\varepsilon} \frac{d q}{d t}$, etc.

\subsection{Local Existence.}

Theorem 5.1 (Local Existence). Consider the initial value problem:

$$
\begin{aligned}
& \psi_{t t}+L \psi=k+\varepsilon j, \\
& -\Delta \tilde{\tilde{a}}_{0}+|\phi|^{2} \tilde{\tilde{a}}_{0}=-2\left(i \tilde{\phi}, \varphi_{\tau}\right)+\varepsilon j_{0}, \\
& -\left(n_{\lambda}, n_{\kappa}\right) \ddot{q}_{\kappa}-\left(n_{\lambda}, \frac{d n_{\kappa}}{d \tau} \dot{q}_{\kappa}\right)-\frac{l}{2}\left(\varphi\left(1-|\phi|^{2}\right), n_{\mu}\right)=-\varepsilon\left(\left(j_{1}, j_{2}, j_{3}\right)^{t}, n_{\mu}\right) \\
& -\varepsilon^{2}\left(\left(\tilde{a}_{t}, \tilde{\phi}_{t}\right)^{t}, \partial_{\tau}^{2} n_{\mu}\right) \\
& -2 \varepsilon\left(\left(\tilde{a}_{t}, \tilde{\phi}_{t}\right)^{t}, \partial_{\tau} n_{\mu}\right)
\end{aligned}
$$

with initial data $\psi(0), \psi_{t}(0), q_{\mu}(0), \dot{q}_{\mu}(0)$ given satisfying the constraint (29), and

$$
|\psi(0)|_{3, a(0)}+\left|\psi_{t}(0)\right|_{2, a(0)}+\left|\dot{q}_{\mu}(0)\right| \leqq \Gamma / 2 .
$$

Then there exist $\varepsilon_{*}(\Gamma), T_{\mathrm{loc}}(\Gamma)$ such that for $\varepsilon<\varepsilon_{*}$ there exists a solution to the initial value problem on the interval $\left[0, T_{\mathrm{loc}}\right]$ satisfying the constraint (29), with the property that $q \in C^{2}\left(0, T_{\mathrm{loc}}\right)$ and the maps

$$
\begin{aligned}
t & \rightarrow\left(\psi, \psi_{t}\right) \in H^{3, a(0)} \oplus H^{2, a(0)}, \\
t & \rightarrow\left(\psi, \psi_{t}\right) \in H^{1, a(0)} \oplus L^{2}, \\
t & \rightarrow \tilde{\tilde{a}}_{0} \in H^{2}
\end{aligned}
$$

are, respectively, strongly continuous, strongly differentiable and strongly differentiable. In addition the solution satisfies the energy identities of Sect. 5.2. 
Proof. See appendix for a simple but non-optimal proof. The proof there is restricted to small $\varepsilon$ because it employs a simple iteration scheme. Presumably this restriction could be removed. However since we are only interested in small $\varepsilon$ we shall not carry this out.

5.2. A Priori Estimate and Proof of Main Theorem. In this section we will prove that the ansatz for the solution presented above does indeed give a good approximation to the solution. In other words we want to consider the local solutions to the coupled system provided by Theorem 5.1, and prove that the $\tilde{\tilde{a}}_{0}, \psi$ are bounded in some norm for a large time - in fact for a time of $O\left(\frac{1}{\varepsilon}\right)$. We also want to know that the highest order modulation equations approximate the real modulation equation - and of course these two problems are coupled together. So we introduce the solution $p_{0}(\tau), q_{0}(\tau)$ to Eqs. (72) with initial data $q_{0}(0), p(0)=g(\dot{q}(0))$ obtained from the initial data for the PDE given above. Next we write the solutions to the full modulation equations as

$$
p(t)=p_{0}(\varepsilon t)+\varepsilon \tilde{p}(t), \quad q(t)=q_{0}(\varepsilon t)+\varepsilon \tilde{q}(t) .
$$

It will be convenient now to write $p, q$ together as $x(t)=(\tilde{p}(t), \tilde{q}(t))$ and make a definition:

$$
v(t, x) \equiv\left(H_{q}\left(p_{0}, q_{0}\right)-H_{q}(p, q), H_{p}(q, p)-H_{p}\left(p_{0}, q_{0}\right)\right)=\varepsilon A x+\varepsilon^{2} \tilde{v}(x),
$$

where $p_{0}(\varepsilon t), q_{0}(\varepsilon t)$ can now be regarded as fixed functions of time. We use the fact that $v$ is smooth to make a Taylor expansion of $v$. With these definitions, our set of equations becomes

$$
\begin{aligned}
\psi_{t t}+L \psi & =k+\varepsilon j, \\
-\Delta \tilde{\tilde{a}_{0}+|\phi|^{2}} \tilde{\tilde{a}}_{0} & =-2\left(i \tilde{\phi}, \varphi_{\tau}\right)+\varepsilon j_{0}, \\
\frac{d x}{d t} & =\varepsilon A(\tau) x+\varepsilon^{2} w\left(t, \psi, \tilde{a}_{0}, x\right),
\end{aligned}
$$

where $w$ represents the eiror terms. This equation will give the necessary estimate for $x$. The main tools for controlling the errors in $\psi$ in our approximation are the energy identities. These are generalisations of the fact that if the background configuration $\alpha, \varphi$ is fixed then the corresponding operator $L$ is time independent, and so the equation

$$
\psi_{t t}+L \psi=0
$$

has a conserved quantity

$$
Q_{1}(t) \equiv \frac{1}{2} \int_{\mathbf{R}^{2}}\left|\psi_{t}\right|^{2}+(\psi, L \psi)=\frac{1}{2}\left|\psi_{t}\right|_{L^{2}}^{2}+\overline{\operatorname{Hess}}_{\alpha, \varphi}(\psi, \psi) .
$$

In our case the background configuration is slowly varying in time, and there are inhomogeneous terms in the equation. However there are still approximate conservation laws due to the fact that the $O(1)$ error terms on the right-hand side are slowly varying in time (see also [Stu92]). We now write down the identities, where $Q_{1}$ is as defined above but with the background configuration understood to be 
slowly varying in time according to Eq. (66). Differentiating we get

$$
\frac{d Q_{1}}{d t}=\left(\psi_{t}, k+\varepsilon j\right)+\varepsilon C(\dot{q}, \psi)
$$

where $C$ represents the terms introduced by the (slow) time variation of the background configuration $a(x ; q(t)), \varphi(x ; q(t))$, and satisfies the estimate

$$
|C(\dot{q}, \psi)| \leqq c|\dot{q}||\psi|_{L^{2}}
$$

We now integrate in time, integrating by parts the $k$ term, and take advantage of the fact that it is slowly varying:

$$
Q_{1}(t)-Q_{1}(0)=[\psi, k]_{0}^{t}-\varepsilon \int_{0}^{t}\left(\left(\psi, k_{\tau}\right)-\left(\psi_{t}, j\right)-C(\dot{q}, \psi)\right) .
$$

We will also need a higher energy in order to estimate the higher Sobolev norms. However we cannot just apply a derivative to the equation as this will introduce error terms which are not slowly varying, so we take advantage of the fact that $L$ gives us equivalent Sobolev information as shown by the results in Sect. 3, and also almost commutes with the equation. Applying $L$ we find

$$
(L \psi)_{t t}+L(L \psi)=L k+\varepsilon L j+2 \varepsilon L_{\tau} \psi_{t}+\varepsilon^{2} L_{\tau \tau} \psi,
$$

from which we deduce an identity corresponding to the previous one for $Q_{2}(t)=$ $\frac{1}{2}\left|(L \psi)_{t}\right|_{L^{2}}^{2}+\overline{\operatorname{Hess}}_{\alpha, \varphi}(L \psi, L \psi)$ : first of all we have

$$
\frac{d Q_{2}}{d t}=\left((L \psi)_{t}, L k+\varepsilon L j+2 \varepsilon L_{\tau} \psi_{t}+\varepsilon^{2} L_{\tau \tau} \psi\right)+\varepsilon C(\dot{q}, L \psi)
$$

and applying the same integration by parts trick we find

$$
\begin{aligned}
Q_{2}(t)-Q_{2}(0)= & {[(L \psi, L k)]_{0}^{t}-\varepsilon \int_{0}^{t}\left(L \psi,(L k)_{\tau}\right)-2\left((L \psi)_{t}, L_{\tau} \psi_{t}\right) } \\
& -\varepsilon \int_{0}^{t}\left((L \psi)_{t}, L_{\tau \tau} \psi+L j\right)-C(\dot{q}, L \psi) .
\end{aligned}
$$

These two identities are the main ingredients needed for the proof of the main theorem. The idea will be to measure the size of $\psi$ with $Q_{1}+Q_{2}$ and apply the Gronwall inequality to deduce boundedness. The estimates for $Q_{1}, Q_{2}$ come from

Lemma 5.2. For the local solutions whose existence is guaranteed by Theorem 5.1 the following inequalities are valid for the corresponding quantities $Q_{1}, Q_{2}$ :

$$
\begin{aligned}
Q_{1}(t) \leqq & Q_{1}(0)+c(|\dot{q}|,|\ddot{q}|)|\psi(t)|_{L^{2}}^{1 / 2}|k(t)|_{L^{2}}^{1 / 2} \\
& +\varepsilon \int_{0}^{t} c\left(|\dot{q}|,|\ddot{q}|,\left|q^{(3)}\right|,|\psi|_{1, a},\left|\psi_{t}\right|_{L^{2}},\left|\tilde{\tilde{a}}_{0}\right|_{H^{1}},\left|\tilde{\tilde{a}}_{0, t}\right|_{H^{1}}\right), \\
Q_{2}(t) \leqq & Q_{2}(0)+c(|\dot{q}|,|\ddot{q}|)|\psi(t)|_{2, a}^{1 / 2}|L k(t)|_{L^{2}}^{1 / 2} \\
& +\varepsilon \int_{0}^{t} c\left(|\dot{q}|,|\ddot{q}|,\left|q^{(3)}\right|,|\psi|_{3, a},\left|\psi_{t}\right|_{2, a},\left|\tilde{\tilde{a}}_{0}\right|_{H^{3}},\left|\tilde{\tilde{a}}_{0, t}\right|_{H^{3}}\right) .
\end{aligned}
$$


In order to make use of this lemma we need to know that $Q_{1}+Q_{2}$ is a good norm, and that $\tilde{\tilde{a}}_{0}$ does not cause any trouble. The next two lemmas address these two issues.

Lemma 5.3. Let $q(t)$ be a differentiable funcion on some interval with $\dot{q}=O(\varepsilon)$. Then on this interval the quantity $Q_{1}+Q_{2}$ is (uniformly) equivalent to $|\psi(t, \cdot)|_{H^{3, a}}+$ $\left|\psi_{t}(t, \cdot)\right|_{H^{2, a}}$. The background connection $a$ is $a(x ; q(t))$.

Proof. To see this, first of all refer to (52), to see that

$$
\begin{aligned}
\left|\psi_{t}\right|_{2, a} & \leqq c\left(\left|\psi_{t}\right|_{L^{2}}+\left|L \psi_{t}\right|_{L^{2}}\right) \\
& \leqq c\left(\left|\psi_{t}\right|_{L^{2}}+\left|(L \psi)_{t}\right|_{L^{2}}+\varepsilon\left|L_{\tau} \psi\right|_{L^{2}}\right. \\
& \leqq c\left(\left|\psi_{t}\right|_{L^{2}}+|\psi|_{L^{2}}+\left|(L \psi)_{t}\right|_{L^{2}}\right) \\
& \leqq c\left(Q_{1}(\psi, \psi)+Q_{2}(\psi, \psi)\right)
\end{aligned}
$$

where we use the assumption on $q$ to bound $\left|L_{\tau} \psi\right|_{L^{2}} \leqq c|\psi|_{L^{2}}$. The result now follows from Corollary 3.5.

Lemma 5.4. For the local solutions in Theorem 5.1 the following bounds hold:

$$
\left|\tilde{\tilde{a}}_{0}\right|_{H^{5}} \leqq c\left(|\psi|_{3, a},\left|\psi_{t}\right|_{2, a}\right), \quad\left|\tilde{\tilde{a}}_{0, t}\right|_{H^{3}} \leqq c\left(|\psi|_{3, a},\left|\psi_{t}\right|_{2}\right) .
$$

Proof. The first one comes from a direct estimate of the equation for $\tilde{\tilde{a}}_{0}$ using the observation in Lemma 9.1 in the appendix on local existence. For the second one has to differentiate the equation for $\tilde{\tilde{a}}_{0}$ and substitute for $\phi_{t t}$, and then estimate for small $\varepsilon$ by taking all $\tilde{\tilde{a}}_{0}$ to the left-hand side. After substituting the equation for $\phi_{t t}$ of course contains second derivatives which is why the second estimate does not have the gain of regularity of the first one.

Lemma 5.5. Consider $\left(\psi, \psi_{t}\right) \in H^{3, a} \oplus H^{2, a}$, and $\left(\tilde{\tilde{a}}_{0}, \tilde{\tilde{a}}_{0, t}\right) \in H^{3} \oplus H^{3}$. Then the error term $j$ satisfies:

$$
|j|_{2, a} \leqq c\left(|\psi|_{3, a},\left|\psi_{t}\right|_{2, a},\left|\tilde{a}_{0}\right|_{3},\left|\tilde{a}_{0}\right|_{3}\right)
$$

Proof. See appendix.

Theorem 5.6 (A Priori Estimate). Consider a local solution as provided by Theorem 5.1. Then if we define

$$
M(t) \equiv \max _{0 \leqq s \leqq t}\left(|\dot{x}(s)|+|\ddot{x}(s)|+Q_{1}(s)+Q_{2}(s)\right)
$$

then there exists a time $T=O\left(\frac{1}{\varepsilon}\right)$ such that for $t<T$,

$$
M^{1 / 2}(t) \leqq c+M^{1 / 2}(0)+\varepsilon \int_{0}^{t} c(M(s)) d s .
$$

Proof. This basically follows from an obvious estimation of the expressions for $Q_{1}, Q_{2}$, using the estimates in the first appendix for the error terms and Lemma 5.4. The only terms this leaves unaccounted for are those involving $\dot{q}, \ddot{q}$ and $q^{(3)}(t)$. To deal with these we notice from the expression in Eq. (74) and Eq. (75) that

$$
|\dot{q}(t)|,|\ddot{q}(t)| \leqq c+\varepsilon \int_{0}^{t} c(M(s)) d s .
$$


Differentiation of Eq. (79) for $\ddot{q}$ then gives, for sufficiently small $\varepsilon$ :

$$
\mid\left(q^{(3)}(t) \mid \leqq c(M(t)) .\right.
$$

This last equation states that $\frac{d^{3} q}{d t^{3}}=O\left(\varepsilon^{3}\right)$ and allows us to estimate $\frac{d k}{d t}$. We insert these expressions into the $Q_{i}$ equations and then just estimate the integrals. This gives

$$
M(t) \leqq M(0)+c\left(M(t)^{1 / 2}+M(0)^{1 / 2}\right)+\varepsilon \int_{0}^{t} c(M(s)) d s,
$$

from which the required estimate follows.

Proof of Main Theorem. We produce a solution for a short time from the local existence theorem. This local solution will satisfy the inequalities needed to prove the a priori estimate just obtained (see appendix). We then apply the Gronwall inequality to the a priori estimate to deduce that for as long as the local solution exists with $M<M_{*}$ :

$$
M(t) \leqq c e^{\varepsilon \int_{0}^{t} c\left(M_{*}\right)} .
$$
From this it is clear that we can continue the local solution for times of $O\left(\frac{1}{\varepsilon}\right)$, by
repeatedly applying the local existence theorem.

\section{Phenomenological Implications and Open Questions}

We see that we have reduced an infinite dimensional system to a finite dimensional one - the Hamiltonian system corresponding to the Hamiltonian

$$
H=\frac{1}{2} g(p, p)+V(q)
$$

where $p$ is the momentum $p_{\mu}=\sum_{\kappa}\left(n_{\mu}, n_{\kappa}\right) \dot{q}_{\kappa}, g$ is the dual metric defined by $g(p, p)=\sum_{\mu, v}\left(n_{\mu}, n_{v}\right) \dot{q}_{\mu} \dot{q}_{v}$, and $V$ is a potential energy defined on the moduli space by

$$
\begin{aligned}
& V(q)=\frac{l}{8} \int_{\mathbf{R}^{2}}\left(1-|\phi|^{2}\right)^{2}=\imath V_{0}(q), \\
& V_{0}(q)=\frac{1}{8} \int_{\mathbf{R}^{2}}\left(1-|\phi|^{2}\right)^{2} .
\end{aligned}
$$

Thus to understand the attraction/repulsion problem for vortices we need to understand how the potential energy $V$ varies with the separation of the two vortices. Notice that since $\lambda=1+i \varepsilon^{2}$ the force changes sign depending on whether $\lambda$ is above or below the critical value, which fits in nicely with the notion that for $\lambda>1$ the force should be repulsive and for $\lambda<1$ attractive. For reasons of symmetry we know that $V$ is only a function of the distance between the vortices

$$
V\left(Z_{1}, Z_{2}\right)=V\left(\left|Z_{1}-Z_{2}\right|\right) \text {. }
$$

Furthermore since we know from Lemma 3.4 that asymptotically the two vortex solution looks like a superposition of single vortices, it follows that $V$ will approach its "two vortex" value asymptotically fast. 
Conjecture. The function $V_{0}$ is a monotonically decreasing function of $\left|Z_{1}-Z_{2}\right|$.

For numerical evidence in favor of this see [JR79].

If this conjecture is true we can deduce that for $\lambda>1$ the vortices repel by looking at the conservation of energy. Consider the motion of two vortices initially at rest equal distances from the origin on the real axis. By translation invariance the centre of mass will remain at rest, so we can choose as co-ordinates

$$
\begin{aligned}
& P(\tau)=Z_{1}(\tau)+Z_{2}(\tau)=0, \\
& Q(\tau)=Z_{1}(\tau) Z_{2}(\tau)=-Z_{1}^{2}(\tau) .
\end{aligned}
$$

We can then write the potential function in terms of $|Q|$ :

$$
v(|Q|)=V_{0}\left(Z_{1},-Z_{1}\right),
$$

and the results of Sect. 2 ensure that $v$ is smooth. Also referring to that section we can write the kinetic energy term as

$$
g(\dot{q}, \dot{q})=\tilde{f}(|Q|)|\dot{Q}|^{2} .
$$

Thus for the present situation conservation of energy takes the form

$$
\frac{1}{2} \tilde{f}(|Q|)|\dot{Q}|^{2}+v(|Q|)=v(|Q(0)|) .
$$

From this we can see that if $l>0$, and if the conjecture is valid, then in the resulting motion we can only have positive kinetic energy if $|Q(\tau)|<|Q(0)|$. Thus the vortices will move away from one another in the $\lambda>1$ case. The same holds true, mutatis mutandis, for attraction when $l<0$. In fact we can be slighty more explicit - write $Q=q_{1}+i q_{2}$, and introduce the conjugate momenta

$$
p_{1}=\tilde{f} \dot{q}_{1} \quad p_{2}=\tilde{f} \dot{q}_{2} .
$$

Using these coordinates the Hamilton equations become

$$
\begin{aligned}
& \frac{d p_{1}}{d \tau}=-\imath \frac{\partial v}{\partial q_{1}}=-\imath \frac{q_{1}}{|Q|} v^{\prime}(|Q|), \\
& \frac{d p_{2}}{d \tau}=-\imath \frac{\partial v}{\partial q_{2}}=-\imath \frac{q_{2}}{|Q|} v^{\prime}(|Q|) .
\end{aligned}
$$

Initial data for the problem under consideration correspond to $q_{1}(0)<0$, $q_{2}(0)=0$. We will clearly have a solution with $q_{2}(\tau)=0$ and $q_{1}(\tau)$ either becoming more or less negative according as $\imath$ is positive or negative. This corresponds to repulsion for $\lambda>1$ and attraction for $\lambda<1$. A more detailed understanding of this should be possible with computer simulations.

Another interesting issue is the right angle scattering phenomenon discussed in the introduction. This has been observed numerically to be robust under departures from the Bogomolny regime (see [SR88, KMR88]). We can now see why this is so - it depends essentially on the fact that $P, Q$ are the appropriate parameters to give a description of the vortices when they are close. In fact using the co-ordinates just introduced we can see this explicitly. If the vortices start close to one another and are given an initial velocity toward one another, we will have initial conditions

$$
q_{1}<0, \quad \dot{q}_{1}=M>0, \quad q_{2}=0, \quad \dot{q}_{2}=0 .
$$


If $M$ is large enough compared with the potential energy then there will be solutions of the equations in which

$$
q_{2}(\tau)=0, \quad q_{1}(\tau)=f(\tau),
$$

where $f$ is a function which changes sign from negative to positive. Changing co-ordinates back to $Z_{1}, Z_{2}$ we have

$$
Z_{1}(\tau)=-Z_{2}(\tau)=\sqrt{-f(\tau)}
$$

which corresponds to right angle scattering. The fact that $\dot{Z}_{1}, \dot{Z}_{2}$ are infinite when $f(\tau)=0$ is a manifestation of the fact that $Z_{1}, Z_{2}$ are not a good co-ordinate system at the point of coincidence $\left(Z_{1}=Z_{2}\right)$. This is not a physical effect. Thus we see that the right angle scattering phenomenon is robust to small changes in $\lambda$, in agreement with the numerical experiments just mentioned. Perhaps the same is true of some of the similar phenomena which occur in monopole scattering in the Bogomolny situation ([AH88]).

Finally we make some comments on the analytical issues raised by this work. The two obvious extensions to make are

(i) To give a similar discussion of monopole scattering. It is possible to give a similar asymptotic discussion of monopole dynamics using the same perturbation techniques (see [Stu]). However the proof given here that those expansions are valid does not immediately go over. The difficulty here lies in the fact that the symmetry is only partially broken - this has the analytical consequence that the Hessian does not contain mass terms at infinity for all the components of $a, \phi-$ only those transverse to the Higgs field. Thus we can say the continuous spectrum touches the origin and so the Hessian is not equivalent to the $H^{1}$ norm orthogonal to the zero modes. Thus new ideas are required to ascertain whether or not the approximation is valid in this case. ${ }^{2}$

(ii) To extend the understanding of vortex dynamics to infinite time intervals. One would hope that as time becomes large the radiation decreases in magnitude pointwise due to dispersive effects, and that the vortices dominate the asymptotic behaviour. This requires a detailed understanding of the effects of dispersion in the linearised problem, which is difficult because there are variable coefficients so the generalised energy methods seem not to be applicable.

\section{Appendix One: The Error Terms}

In this appendix we give the error terms which were not explicitly given in Sect. 3:

$$
\begin{aligned}
& j_{0}=-\varepsilon\left(\tilde{\phi}, \partial_{t} \tilde{\phi}\right)-2 \varepsilon^{2}(\varphi, \tilde{\phi}) \tilde{a}_{0}-\varepsilon^{4}|\tilde{\phi}|^{2} \tilde{\tilde{a}}_{0}, \\
& j_{i}=\varepsilon\left(i \tilde{\phi}, d_{i}^{(0)} \tilde{\phi}\right)+\varepsilon\left(\varphi, \tilde{a}_{i} \tilde{\phi}\right)+\varepsilon^{3}\left(\tilde{\phi}, \tilde{a}_{i} \tilde{\phi}\right)+\partial_{t} \partial_{i} \tilde{\tilde{a}}_{0}, \\
& j_{3}=\frac{l \varepsilon^{2}}{2}\left(\tilde{\phi}\left(1-2|\phi|^{2}\right)-\varphi^{2} \tilde{\bar{\phi}}\right)-\lambda \varepsilon^{2}\left(\varphi|\tilde{\phi}|^{2}-\bar{\varphi} \tilde{\phi}^{2}\right)
\end{aligned}
$$

2 This has since been successfully carried out in [D.S] 
Dynamics of Abelian Higgs Vortices in Near Bogomolny Regime

$$
\begin{aligned}
& -\lambda \varepsilon^{4} \overline{\tilde{\phi}} \tilde{\phi}^{2}-\varepsilon^{2}\left(\tilde{a}_{i}^{2} \varphi+i \tilde{a}_{i} d_{i}^{(0)} \tilde{\phi}-i \partial_{i}\left(\tilde{a}_{i} \tilde{\phi}\right)\right) \\
& -\varepsilon^{4} \tilde{a}_{i}^{2} \tilde{\phi}+i \varepsilon \frac{\partial \tilde{a}_{0}}{\partial t} \varphi+2 i \varepsilon^{2} \tilde{a}_{0} \partial_{\tau} \varphi+i \varepsilon^{3} \partial_{t}\left(\tilde{\tilde{a}_{0}} \tilde{\phi}\right) \\
& +i \varepsilon^{3} \tilde{\tilde{a}}_{0} \frac{\partial \tilde{\phi}}{\partial t}+i \varepsilon^{4} \tilde{\tilde{a}}_{0}^{2} \varphi+i \varepsilon^{6} \tilde{\tilde{a}}_{0}^{2} \tilde{\phi} .
\end{aligned}
$$

We now assume that all functions are in the spaces used in the text:

$$
\psi \in H^{3, a}, \quad \psi_{t} \in H^{2, a}, \quad \tilde{\tilde{a}}_{0} \in H^{4}, \quad \tilde{\tilde{a}}_{0, t} \in H^{3},
$$

then the functions are all in $L^{\infty}$ and we have the following estimates:

$$
\begin{aligned}
\left|j_{0}\right|_{r} & \leqq c\left(|\psi|_{r, a},\left|\psi_{t}\right|_{r, a},\left|\tilde{\tilde{a}}_{0}\right|_{r}\right), \\
\left|j_{i}\right|_{r} & \leqq c\left(|\psi|_{r+1, a}+\left|\tilde{\tilde{a}}_{0, t}\right|_{r+1}\right) \\
\left|j_{3}\right|_{r, a} & \leqq c\left(|\psi|_{r+1, a},\left|\psi_{t}\right|_{r, a},\left|\tilde{\tilde{a}}_{0}\right|_{r+1},\left|\tilde{\tilde{a}}_{0, t}\right|_{r}\right)
\end{aligned}
$$

valid for $r \geqq 2$, where $c(\cdot, \ldots, \cdot)$ indicates an increasing positive function of several arguments as in the text.

\section{Appendix Two: The Index Calculation}

In this appendix we give a mathematical version of the index calculation of [Wei79]. We are interested in the operator $\mathscr{D}$ defined in (30) which can be written out more fully as

$$
\mathscr{D}=\frac{1}{2}\left(\begin{array}{cccc}
\partial_{1} & \partial_{2} & \phi_{2} & -\phi_{1} \\
-\partial_{2} & \partial_{1} & \phi_{1} & \phi_{2} \\
\phi_{2} & \phi_{1} & \partial_{1}+A_{2} & -\partial_{2}+A_{1} \\
-\phi_{1} & \phi_{2} & \partial_{2}-A_{1} & \partial_{1}+A_{2}
\end{array}\right)
$$

while its adjoint is given by

$$
\mathscr{D}^{*}=\frac{1}{2}\left(\begin{array}{rrcc}
-\partial_{1} & \partial_{2} & \phi_{2} & -\phi_{1} \\
-\partial_{2} & -\partial_{1} & \phi_{1} & \phi_{2} \\
\phi_{2} & \phi_{1} & -\partial_{1}+A_{2} & \partial_{2}+A_{1} \\
-\phi_{1} & \phi_{2} & -\partial_{2}-A_{1} & -\partial_{1}+A_{2}
\end{array}\right) .
$$

We first of all prove that these are Fredholm:

Lemma 8.1. Consider the operators $\mathscr{D}, \mathscr{D}^{*}$ between the spaces

$$
\mathscr{D}, \mathscr{D}^{*}:\left(H^{1}\right)^{4} \rightarrow\left(L^{2}\right)^{4} \text {. }
$$

They are Fredholm.

Proof. We will use the fact that from [Hor79, Tay71, BS78] that an operator of the given form is Fredholm if the following two conditions are satisfied: (i) The coefficients satisfy the property

$$
\left.\left|\partial_{x}^{\alpha}\right| a|\leqq c| x\right|^{-|\alpha|}
$$


which is certainly true from (26). (ii) In addition if we look at the asymptotic behaviour in phase space then the symbols are non-singular on sufficiently large spheres.

To see this write out the symbols:

$$
\begin{gathered}
\sigma(x, \xi) \equiv \frac{1}{2}\left(\begin{array}{rrcc}
\xi_{2} & -\xi_{1} & \phi_{2} & -\phi_{1} \\
\xi_{1} & \xi_{2} & \phi_{1} & \phi_{2} \\
\phi_{2} & \phi_{1} & -\xi_{2}+A_{2} & -\xi_{1}+A_{1} \\
-\phi_{1} & \phi_{2} & \xi_{1}-A_{1} & -\xi_{2}+A_{2}
\end{array}\right), \\
\sigma^{*}(x, \xi) \equiv \frac{1}{2}\left(\begin{array}{rrcc}
\xi_{2} & +\xi_{1} & \phi_{2} & -\phi_{1} \\
-\xi_{1} & \xi_{2} & \phi_{1} & \phi_{2} \\
\phi_{2} & \phi_{1} & -\xi_{2}+A_{2} & +\xi_{1}-A_{1} \\
-\phi_{1} & \phi_{2} & -\xi_{1}+A_{1} & -\xi_{2}+A_{2}
\end{array}\right) .
\end{gathered}
$$

We wish to show these are non-singular on sufficiently large spheres

$$
|x|^{2}+|\xi|^{2}=R^{2} .
$$

Thus either $|x|^{2}$ or $|\xi|^{2}$ is bigger than $\frac{1}{2} R^{2}$ - in the former case one can calculate that the asymptotic determinant is $\left(1+|\xi|^{2}\right)^{2}(1+o(1))$ so it is non-singular in this case. In the case $|\xi|^{2}$ is large, the determinant is $|\xi|^{4}(1+o(1))$, and so in both cases it is non-singular. Thus by the criterion above we see that $\mathscr{D}$ is Fredholm, and so is its adjoint.

Lemma 8.2. Ind $\mathscr{D}=2 N$ and $\operatorname{Ker} \mathscr{D}^{*}=\emptyset$.

Proof. We would like to apply the formula of Fedosov ([Fed74, BS78]) to calculate this index directly. Fedosov's formula says the index is given by

$$
\text { Ind } \mathscr{D}=-\left(\frac{i}{2 \pi}\right)^{2} \frac{1}{3 !} \int_{S_{R}^{3}} \operatorname{Tr}_{\mathrm{C}}\left(\sigma^{-1} d \sigma\right)^{3} .
$$

This is not yet in a form convenient for calculation - we shall take the limit $|x| \rightarrow \infty$, so that by (26):

$$
\begin{gathered}
\phi_{1} \rightarrow \cos N \theta+O\left(e^{-\delta|x|}\right), \quad \phi_{2} \rightarrow \sin N \theta+O\left(e^{-\delta|x|}\right), \\
A_{1}, A_{2}=O\left(\frac{1}{|x|}\right),
\end{gathered}
$$

and consider the operator

$$
\mathscr{D}_{\infty}=\frac{1}{2}\left(\begin{array}{cccc}
\partial_{1} & \partial_{2} & \sin N \theta & -\cos N \theta \\
-\partial_{2} & \partial_{1} & \cos N \theta & \sin N \theta \\
\sin N \theta & \cos N \theta & \partial_{1} & -\partial_{2} \\
-\cos N \theta & \sin N \theta & \partial_{2} & \partial_{1}
\end{array}\right)
$$


with corresponding symbol

$$
\sigma_{\infty}(x, \xi) \equiv \frac{1}{2}\left(\begin{array}{cccc}
\xi_{2} & -\xi_{1} & \sin N \theta & -\cos N \theta \\
\xi_{1} & \xi_{2} & \cos N \theta & \sin N \theta \\
\sin N \theta & \cos N \theta & -\xi_{2} & -\xi_{1} \\
-\cos N \theta & \sin N \theta & \xi_{1} & -\xi_{2}
\end{array}\right) .
$$

We will use the following result

Lemma 8.3. The operator $\mathscr{D}_{\infty}$ is Fredholm and

$$
\text { Ind } \mathscr{D}_{\infty}=\text { Ind } \mathscr{D} \text {. }
$$

Proof. We use the result in Theorem 5.26 of chapter four of [Kat66] which states that a relatively compact perturbation of a Fredholm operator is also Fredholm with the same index. To see that $\mathscr{D}_{\infty}$ is a relatively compact perturbation of $\mathscr{D}$ we notice that the matrix $B=\mathscr{D}-\mathscr{D}_{\infty}$ has entries which are $O\left(\frac{1}{|x|}\right)$ for large $x$. Now consider a sequence $\psi^{i}$ bounded in $H^{1}$ - we have to find a strongly $L^{2}$ convergent subsequence to $B \psi^{i}$ (see p. 194 of the same reference). We have a weak limit $\psi^{i} \rightarrow \psi \in H^{1}$ by Banach-Aloagau, so we must show that $\left|B\left(\psi^{i}-\psi\right)\right|_{L^{2}} \rightarrow 0$. To see that this is so we split up the integral using the characteristic function $\chi$ of a ball of radius $r$ :

$$
\left|B\left(\psi^{i}-\psi\right)\right|_{L^{2}}=\left|B \chi\left(\psi^{i}-\psi\right)\right|_{L^{2}}+\left|B(1-\chi)\left(\psi^{i}-\psi\right)\right|_{L^{2}}
$$

By choosing $r$ large we can make the second term less than $\varepsilon / 2$, and then make $i$ so that the first term is also $<\varepsilon / 2$ by Rellich. This completes the proof.

Thus we now need to calculate

$$
\text { Ind } \mathscr{D}_{\infty}=-\left(\frac{i}{2 \pi}\right)^{2} \frac{1}{3 !} \int_{S_{R}^{3}} \operatorname{Tr}_{\mathbf{C}}\left(\sigma_{\infty}^{-1} d \sigma_{\infty}\right)^{3} .
$$

The integrand can be evaluated directly using a symbolic manipulator, and one finds

$$
\operatorname{Tr}_{\mathbf{C}}\left(\sigma_{\infty}^{-1} d \sigma_{\infty}\right)^{3}=\frac{12 N s}{\left(1+s^{2}\right)^{2}} d \xi_{1} d \xi_{2} d \theta
$$

where $|\xi|^{2}=s^{2}$, so that the integral becomes

$$
\text { Ind } \begin{aligned}
\mathscr{D} & =(4 \pi)^{-2} \int_{0}^{2 \pi} \int_{0}^{2 \pi} \int_{0}^{R} \frac{2 s}{\left(1+s^{2}\right)^{2}} d s d \theta d \phi \\
& =2 N\left[\frac{-1}{\left(1+s^{2}\right)}\right]_{0}^{R}=2 N .
\end{aligned}
$$

Next we prove the second result i.e. $\operatorname{Ker} \mathscr{D}^{*}=\emptyset$. To see this we assume that $(\beta, \psi)$ is a square integrable element of the kernel of $\mathscr{D}^{*}$ - it will then automatically be smooth, and will satisfy the equations

$$
\begin{aligned}
-\bar{\partial} \beta+i \bar{\phi} \psi & =0 \\
-\partial \psi+i \alpha \psi-\frac{i}{4} \phi \beta & =0 .
\end{aligned}
$$


Operate $\partial$ on the first equation, and substitute for $\partial \psi$ from the second equation, then with use of the Bogomolny equation $\partial \bar{\phi}+i \alpha \bar{\phi}=0$ we find

$$
-\partial \bar{\partial} \beta+|\phi|^{2} / 4 \beta=0
$$

from which we deduce $\beta=0$ and hence from the first equation $\psi=0$ as required. This completes the proof of the whole lemma.

\section{Appendix Three: The Local Existence Theorem}

In this appendix we prove the local existence Theorem 5.1 by an iteration procedure. We will often suppress the index $\mu$ as it is not important, and we will write $c(\Gamma)$ for a variable constant depending on $\Gamma$. The first step is to rewrite the equations in a way suitable for this - we have to

(i) First of all solve the modulation equation for $\ddot{q}_{\mu}$, which is possible for sufficiently small $\varepsilon$ on account of the uniform invertibility of the metric discussed in Sect. 2.2. This leads to an equation of the form

$$
\ddot{q}_{\mu}=f_{2}\left(\psi, \psi_{t}, q, q_{t}, \tilde{\tilde{a}}_{0}, \tilde{\tilde{a}}_{0, t}\right) .
$$

(ii) Take the formula for $\ddot{q}$ from (i) and substitute this into the equation for $\psi$ leading to an equation of the form:

$$
\frac{\partial^{2} \psi}{\partial t^{2}}+L_{0} \psi=f_{1}\left(\psi, \psi_{t}, \tilde{a}_{0}, \tilde{a}_{0, t}, q, \dot{q}\right),
$$

where $L_{0}$ is the operator defined in Eq. (48). We will now produce iterates according to the scheme:

$$
\begin{aligned}
O^{(i)} \tilde{\tilde{a}}_{0}^{(i+1)} & =f_{0}\left(\psi^{(i)}, \psi_{t}^{(i)}, q, \dot{q}\right)=-2\left(i \tilde{\phi}, \phi_{\tau}\right)-\varepsilon\left(i \tilde{\phi}^{(i)}, \partial_{t} \tilde{\phi}^{(i)}\right), \\
\frac{\partial^{2} \psi^{(i+1)}}{\partial t^{2}}+L_{0} \psi^{(i+1)} & =f_{1}\left(\psi^{(i)}, \psi_{t}^{(i)}, \tilde{\tilde{a}}_{0}^{(i+1)}, \tilde{\tilde{a}}_{0, t}^{(i+1)}, q^{(i)}, \dot{q}^{(i)}\right), \\
\ddot{q}_{\mu}^{(i+1)} & =f_{2}\left(\psi^{(i)}, \psi_{t}^{(i)}, q^{(i)}, q_{t}^{(i)}, \tilde{\tilde{a}}_{0}^{(i+1)}, \tilde{\tilde{a}}_{0, t}^{(i+1)}\right),
\end{aligned}
$$

where the operator $O^{(i)}$ is defined by

$$
O^{(i)} u=-\Delta u+\left|\phi^{(i)}\right|^{2} u+2 \varepsilon^{2}\left(\phi^{(i)}, \tilde{\phi}^{(i)}\right) u+\varepsilon^{4}\left|\tilde{\phi}^{(i)}\right|^{2} u
$$

with $\phi^{(i)}=\phi\left(x ; q^{(i)}(t)\right)$. We will take as initial data for $q$ the $q(0), \dot{q}(0)$. For $\psi$ we shall take initial data $\psi^{(i)}(0), \psi_{t}^{(i)}(0)$ obtained by smoothing and introducing a cutoff at spatial infinity in a fashion which disappears as $i \rightarrow \infty$ :

$$
\left|\psi^{(i)}(0)-\psi(0)\right|_{3, a(0)} \leqq \delta 2^{-i}
$$

This is just a technical device to ensure that all formal manipulations are allowed in the following calculations, since the iterates will be smooth and exponentially decaying at spatial infinity (see also Corollary 9.2 below). It also allows one to prove that the local solution satisfies the identities for $Q_{1}, Q_{2}$ in Sect. 5 by taking the limit of the corresponding identities for the iterates. The smoothed initial values can be used as the first iterates. We will use the norms:

$$
\left\|\tilde{\tilde{a}}_{0}(t)\right\| \equiv \max _{[0, t]}\left(\left|\tilde{\tilde{a}}_{0}\right|_{H^{4}}+\left|\tilde{\tilde{a}}_{0, t}\right|_{H^{3}}\right),
$$


and

$$
\|\psi(t)\| \equiv \max _{[0, t]}\left(|\psi|_{3, a(0)}+\left|\psi_{t}\right|_{2, a(0)}\right)
$$

where the $a(0)$ refers to the background connection as in Sect. 3. We will assume that the initial data satisfy

$$
\left\|\psi^{(i)}\right\|,\left|q^{(i)}\right| \leqq \Gamma / 2
$$

and aim to produce iterates within the space

$$
\left\|\psi^{(i)}\right\|,\left|q^{(i)}\right| \leqq \Gamma
$$

Lemma 9.1. Let $\left\|\psi^{(i)}\right\| \leqq \Gamma$, then there exists $\varepsilon_{0}(\Gamma)$ such that for $\varepsilon<\varepsilon_{1}(\Gamma)$ we have

$$
c(\Gamma)^{-1}|u|_{H^{1}} \leqq\left(u, O^{(i)} u\right) \leqq c(\Gamma)|u|_{H^{1}} .
$$

Proof. This is certainly true for the quadratic form defined by $-\Delta+\left|\phi^{(i)}\right|^{2}$, and so subject to the restriction in the theorem:

$$
\left(u, O^{(i)} u\right) \leqq\left(u,-\Delta u+\left|\phi^{(i)}\right|^{2} u\right)+\varepsilon^{2} c(\Gamma)|u|_{L^{2}}
$$

which gives the result immediately.

Corollary 9.2. Each iterate $\tilde{\tilde{a}}_{0}^{(i)}$ is exponentially decaying in space.

Proof. See e.g. [JT82, Sect. 3.7]

We now see that the iterates are all well defined and using the error bounds in the appendix satisfy the basic estimates. For $\varepsilon<\varepsilon_{1}(\Gamma)$ :

$$
\left|\tilde{\tilde{a}}_{0}^{(i+1)}\right|_{H^{r+2}} \leqq c(\Gamma)\left|f_{0}\right|_{H^{r}},
$$

$$
\begin{gathered}
\max _{[0, T]}\left(\left|\psi^{(i+1)}\right|_{r+1, a(0)}+\left|\psi_{t}^{(i+1)}\right|_{r, a(0)} \leqq\left|\psi^{(i+1)}(0)\right|_{r+1, a(0)}+\left|\psi_{t}(0)\right|_{r, a(0)}+\int_{0}^{T}\left|f_{1}\right|_{r} d t,\right. \\
\max _{[0, T]}\left|\dot{q}^{(i+1)}\right| \leqq|\dot{q}(0)|+\int_{0}^{T}\left|f_{2}\right| d t .
\end{gathered}
$$

We are going to obtain a sequence convergent with respect to the norm

$$
\left\|\psi\left(T_{\mathrm{loc}}\right)\right\|+\left\|\tilde{\tilde{a}}_{0}\left(T_{\mathrm{loc}}\right)\right\|+\max _{0 \leqq t \leqq T_{\mathrm{loc}}}\left(\left|\psi_{t t}\right|_{1, a(0)}+\sum_{\mu}\left|\dot{q}_{\mu}\right|\right) .
$$

Applying Eq. (80), we see that for $\varepsilon<\varepsilon_{1}(\Gamma)$ small we have the following estimates for some numbers $A(\Gamma), B(\Gamma)$ :

$$
\begin{aligned}
&\left\|\tilde{\tilde{a}}_{0}^{(i+1)}\right\| \leqq A(\Gamma)+\varepsilon c(\Gamma)\left|\psi_{t t}^{(i)}\right|_{1, a(0)}, \\
&\left\|\psi^{(i+1)}\right\| \leqq \Gamma / 2+\int_{0}^{T} c(\Gamma)\left(1+\varepsilon\left\|\tilde{\tilde{a}}_{0}^{(i+1)}\right\|\right) d t, \\
&\left|\psi_{t t}^{(i+1)}\right|_{1, a(0)} \leqq B(\Gamma)\left(1+\varepsilon\left\|\tilde{\tilde{a}}_{0}^{(i+1)}\right\|\right), \\
&\left|\dot{q}^{(i+1)}\right| \leqq \Gamma / 2+\int_{0}^{T} c(\Gamma)\left(1+\varepsilon\left\|\tilde{\tilde{a}}_{0}^{(i+1)}\right\|\right) d t .
\end{aligned}
$$

The difficult term $\psi_{t t}$ appears because we have to estimate $\left\|\tilde{\tilde{a}}_{0, t}\right\|$. We now see that 
Claim. There exist $\varepsilon_{2}(\Gamma), T_{1}(\Gamma)$ such that for $\varepsilon<\varepsilon_{2}, t<T_{1}$ the iterates are uniformly bounded in the space:

$$
\begin{aligned}
X\left(T_{1}\right)= & \left\{\left(\tilde{\tilde{a}}_{0}, \psi, q\right):\left\|\psi\left(T_{1}\right)\right\|, \max _{[0, T]}|q| \leqq \Gamma,\left\|\tilde{\tilde{a}}_{0}\left(T_{1}\right)\right\| \leqq 2 A(\Gamma),\right. \\
& \left.\max _{[0, T]}\left|\psi_{t t}\right|_{1, a(0)} \leqq 2 B(\Gamma)\right\} .
\end{aligned}
$$

Having done this we use the following difference estimates to get a contraction in the norm mentioned above, using a two step induction. First of all we see that

$$
\left\|\psi^{(i+1)}-\psi^{(i)}\right\| \leqq \delta 2^{-1}+c(\Gamma) \int\left(\left\|\psi^{(i)}-\psi^{(i-1)}\right\|+\left\|\tilde{\tilde{a}}_{0}^{(i+1)}-\tilde{\tilde{a}}_{0}^{i}\right\|\right) d t .
$$

This is then used in the corresponding $\tilde{\tilde{a}}_{0}$ equation to give:

$$
\begin{aligned}
\left\|\tilde{\tilde{a}}_{0}^{(i+1)}-\tilde{\tilde{a}}_{0}^{(i)}\right\| \leqq & c(\Gamma) \int\left(\left\|\psi^{(i)}-\psi^{(i-1)}\right\|+\left\|\tilde{\tilde{a}}_{0}^{(i+1)}-\tilde{\tilde{a}}_{0}^{(i)}\right\|\right) d t \\
& +\varepsilon c(\Gamma)\left|\psi_{t t}^{(i)}-\psi_{t t}^{(i-1)}\right| \\
\left|\psi_{t t}^{(i+1)}-\psi_{t t}^{(i)}\right|_{1, a(0)} \leqq & c(\Gamma)\left(\left\|\psi^{(i+1)}-\psi^{(i)}\right\|+\left\|\psi^{(i)}-\psi^{(i-1)}\right\|\right. \\
& \left.+\left|\dot{q}^{(i)}-\dot{q}^{(i-1)}\right|\right)+\varepsilon c(\Gamma)\left\|\tilde{\tilde{a}}_{0}^{(i+1)}-\tilde{\tilde{a}}_{0}^{(i)}\right\| .
\end{aligned}
$$

We now define

$$
\begin{aligned}
Q^{(i)}= & \mid \psi^{(i+1)}-\psi^{(i)}\|+\| \tilde{\tilde{a}}_{0}^{(i+1)}-\tilde{\tilde{a}}_{0}^{(i)} \| \\
& +\max _{0 \leqq t \leqq T_{\mathrm{loc}}}\left(\| \psi_{t t}^{(i+1)}-\left.\psi_{t t}^{(i)}\right|_{1, a(0)}+\sum_{\mu}\left|\dot{q}_{\mu}^{(i+1)}-\dot{q}_{\mu}^{(i)}\right|\right) .
\end{aligned}
$$

Claim. Given $r<1$ there exists $\varepsilon_{3}, T_{2}$ such that for $\varepsilon<\varepsilon_{3}, t<T_{2}$ we have

$$
Q^{j} \leqq r\left(Q^{(j-1)}+Q^{(j-2)}\right)+\delta 2^{-j}
$$

From this it follows that

$$
\sum_{j=1}^{\infty} Q_{j}<\infty
$$

for sufficiently small $r$, which shows that we have a convergent sequence in $X\left(T_{\text {loc }}\right)$ for small enough $T_{\text {loc }}$. The convergence being uniform we get the continuity properties of the map states in the theorem. To see the differentiability properties we use the fact that uniform bounds on $\left|\psi_{t}\right|_{2}$ imply from the equation uniform bounds on $\left|\psi_{t t t}\right|_{L^{2}}$ and so map $t \rightarrow\left(\psi, \psi_{t}\right) \in H^{1} \oplus L^{2}$ is strongly differentiable. Notice also that $\tilde{\tilde{a}}_{0, t}^{(i)}$ are uniformly bounded in $H^{3}$, and hence $t \rightarrow \widetilde{\tilde{a}}_{0} \in H^{3}$ is continuous, while the uniform boundedness of $\tilde{\tilde{a}}_{0, t t}^{(i)}$ in $H^{2}$ ensures that $t \rightarrow \tilde{\tilde{a}}_{0} \in H^{2}$ is differentiable.

\section{References}

[AH88] Atiyah, M., Hitchin, N.: Geometry and Dynamics of Magnetic Monopoles. Princeton N.J.: Princeton University Press 1988

[Ben72] Benjamin, T.B.: Stability of solitons. Proc. Royal Soc. A328, 153-179 (1972)

[BS78] Bott, R., Seeley, R.: Some remarks on the paper of Callias. Commun. Math. Phys. 62, 235 (1978) 
[DHW82] Perez, F., Henry, D., Wrezinski, W.: Stability theory for solitary wave solutions of scalar field equations. Commun. Math. Phys. 85, 351-361 (1982)

[D.S] Stuart, D.: The geodesic approximation for monopoles. Preprint

[FED74] Fedosov, B.: Analytic formulae for index of elliptic operators. Trans. Mosc. Math. Soc. 30, 159-240 (1974)

[Hit88] Hitchin, N.: Geometry and topology of moduli spaces. Springer Lecture Notes in Mathematics 1451, 1-48 (1988)

[Hor79] Hormander, L.: Weyl calculus of pseudo differential operators. Commun. Pure Applied Math. 32, 359-443 (1979)

[JR79] Jacobs, L., Rebbi, C.: Interaction of superconducting vortices. Phys. Rev. B 19, 4486-4494 (1979)

[JT82] Jaffe, A., Taubes, C.: Vortices and Monopoles. Boston, Mass.: Birkhauser 1982

[Kat66] Kato, T.: Perturbation Theory for Linear Operators. Berlin, Heidelberg, New York: Springer 1966

[KMR88] Meyers, E., Moriaty, K., Rebbi, C.: Dynamical interactions of cosmic strings and flux vortices. Phys. Lett. B 207 (1988)

[Man82] Manton, N.: A remark on scattering of BPS monopoles. Phys. Lett. 110B, 54-56 (1982)

[MS78] McLaughlin, D., Scott, A.: Perturbation analysis of fluxon dynamics. Phys. Rev. A 18, 1652-1677 (1978)

[Plo80] Plohr, B.: Existence, Regularity and Behaviour of Isotropic Solutions of Classical Gauge Field Theories. Ph.D. thesis, Princeton University, 1980

[Sam92] Samols, T.: Vortex scattering. Commun. Math. Phys. 145, 149-179 (1992)

[SR88] Shellard, E., Ruback, P.: Vortex scattering in two dimensions. Phys. Lett. B 209, 262-270 (1988)

[Stu] Stuart, D.: Solitary wave perturbation theory in the presence of gauge symmetry. Preprint

[Stu92] Stuart, D.: Perturbation theory for kinks. Commun. Math. Phys. 149, 433-462 (1992)

[Tau82] Taubes, C.: Existence of a non-minimal solution to Yang-Mills-Higgs equations. Commun. Math. Phys. 86, 257-320 (1982)

[Tay71] Taylor, M.: Gelfand theory of pseudo-differential operators and hypoelliptic operators. Trans. Am. Math. Soc. 153, 495-510 (1971)

[Wei79] Weinberg, E.: Multivortex solutions of the Ginzburg-Landau solutions. Phys. Rev. D 19(10), 3008-3012 (1979)

[Wit77] Witten, E.: Some exact multipseudoparticle solutions of classical Yang-Mills theory. Phys. Rev. Lett. 38, 121-124 (1977)

Communicated by A. Jaffe 
